\title{
RIESZ BASES OF WAVELETS AND APPLICATIONS TO NUMERICAL SOLUTIONS OF ELLIPTIC EQUATIONS
}

\author{
RONG-QING JIA AND WEI ZHAO
}

\begin{abstract}
We investigate Riesz bases of wavelets in Sobolev spaces and their applications to numerical solutions of the biharmonic equation and general elliptic equations of fourth-order.

First, we study bicubic splines on the unit square with homogeneous boundary conditions. The approximation properties of these cubic splines are established and applied to convergence analysis of the finite element method for the biharmonic equation. Second, we develop a fairly general theory for Riesz bases of Hilbert spaces equipped with induced norms. Under the guidance of the general theory, we are able to construct wavelet bases for Sobolev spaces on the unit square. The condition numbers of the stiffness matrices associated with the wavelet bases are relatively small and uniformly bounded. Third, we provide several numerical examples to show that the numerical schemes based on our wavelet bases are very efficient. Finally, we extend our study to general elliptic equations of fourth-order and demonstrate that our numerical schemes also have superb performance in the general case.
\end{abstract}

\section{INTRODUCTION}

In this paper, we investigate Riesz bases of wavelets in Sobolev spaces and their applications to numerical solutions of the biharmonic equation and general elliptic equations of fourth-order.

We use $\mathbb{N}, \mathbb{Z}, \mathbb{R}$, and $\mathbb{C}$ to denote the set of positive integers, integers, real numbers, and complex numbers, respectively. For $s \in \mathbb{N}$, we use $\mathbb{R}^{s}$ to denote the $s$-dimensional Euclidean space with the inner product of $x=\left(x_{1}, \ldots, x_{s}\right)$ and $y=\left(y_{1}, \ldots, y_{s}\right)$ in $\mathbb{R}^{s}$ given by $x \cdot y:=x_{1} y_{1}+\cdots+x_{s} y_{s}$.

Let $\mathbb{N}_{0}:=\mathbb{N} \cup\{0\}$. An element of $\mathbb{N}_{0}^{s}$ is called a multi-index. The length of a multi-index $\mu=\left(\mu_{1}, \ldots, \mu_{s}\right) \in \mathbb{N}_{0}^{s}$ is given by $|\mu|:=\mu_{1}+\cdots+\mu_{s}$. For $\mu=\left(\mu_{1}, \ldots, \mu_{s}\right) \in \mathbb{N}_{0}^{s}$ and $x=\left(x_{1}, \ldots, x_{s}\right) \in \mathbb{R}^{s}$, define $x^{\mu}:=x_{1}^{\mu_{1}} \cdots x_{s}^{\mu_{s}}$. A polynomial is a finite sum of the form $\sum_{\mu} c_{\mu} x^{\mu}$ with $c_{\mu}$ being complex numbers. The degree of a polynomial $q=\sum_{\mu} c_{\mu} x^{\mu}$ is defined to be $\operatorname{deg} q:=\max \left\{|\mu|: c_{\mu} \neq 0\right\}$. By $\Pi_{k}$ we denote the linear space of all polynomials of degree at most $k$.

Received by the editor March 17, 2009 and, May 13, 2010.

2010 Mathematics Subject Classification. Primary 42C40, 65N30, 41A15, 41A25, 46E35.

Key words and phrases. Riesz bases, multilevel decompositions, splines, wavelets, wavelets on bounded domains, rate of convergence, biharmonic equation, elliptic equations.

The authors were supported in part by NSERC Canada under Grant OGP 121336.

(C)2011 American Mathematical Society Reverts to public domain 28 years from publication 
Let $\Omega$ be a (Lebesgue) measurable subset of $\mathbb{R}^{s}$. Suppose $f$ is a complex-valued (Lebesgue) measurable function on $\Omega$. For $1 \leq p<\infty$, let

$$
\|f\|_{p, \Omega}:=\left(\int_{\Omega}|f(x)|^{p} d x\right)^{1 / p}
$$

and let $\|f\|_{\infty, \Omega}$ denote the essential supremum of $|f|$ on $\Omega$. When $\Omega=\mathbb{R}^{s}$, we omit the reference to $\mathbb{R}^{s}$. For $1 \leq p \leq \infty$, by $L_{p}(\Omega)$ we denote the Banach space of all measurable functions $f$ on $\Omega$ such that $\|f\|_{p, \Omega}<\infty$. Equipped with the norm $\|\cdot\|_{p, \Omega}, L_{p}(\Omega)$ becomes a Banach space. For $p=2, L_{2}(\Omega)$ is a Hilbert space with the inner product given by $\langle f, g\rangle:=\int_{\Omega} f(x) \overline{g(x)} d x, f, g \in L_{2}(\Omega)$. Let $\mathcal{E}_{\Omega}$ be the extension operator that maps each function $f$ on $\Omega$ to the function $\tilde{f}$ on $\mathbb{R}^{s}$ given by $\tilde{f}(x):=f(x)$ for $x \in \Omega$ and $\tilde{f}(x):=0$ for $x \in \mathbb{R}^{s} \backslash \Omega$. Then $\mathcal{E}_{\Omega}$ is an embedding from $L_{p}(\Omega)$ to $L_{p}\left(\mathbb{R}^{s}\right)$. We may identify $L_{p}(\Omega)$ with $\mathcal{E}_{\Omega}\left(L_{p}(\Omega)\right)$. Thus we may view $L_{p}(\Omega)$ as the subspace of $L_{p}\left(\mathbb{R}^{s}\right)$ consisting of all functions in $L_{p}\left(\mathbb{R}^{s}\right)$ that vanish outside $\Omega$.

For a vector $y=\left(y_{1}, \ldots, y_{s}\right) \in \mathbb{R}^{s}$, let $D_{y}$ denote the differential operator given by

$$
D_{y} f(x):=\lim _{t \rightarrow 0} \frac{f(x+t y)-f(x)}{t}, \quad x \in \mathbb{R}^{s} .
$$

Moreover, we use $\nabla_{y}$ to denote the difference operator given by $\nabla_{y} f=f-f(\cdot-y)$. Let $e_{1}, \ldots, e_{s}$ be the unit coordinate vectors in $\mathbb{R}^{s}$. For $j=1, \ldots, s$, we write $D_{j}$ for $D_{e_{j}}$. For a multi-index $\mu=\left(\mu_{1}, \ldots, \mu_{s}\right)$, we use $D^{\mu}$ to denote the differential operator $D_{1}^{\mu_{1}} \cdots D_{s}^{\mu_{s}}$.

Suppose $\Omega$ is a (nonempty) open subset of $\mathbb{R}^{s}$. Let $C(\Omega)$ be the linear space of all continuous functions on $\Omega$. By $C_{c}(\Omega)$ we denote the linear space of all continuous functions on $\Omega$ with compact support contained in $\Omega$. For an integer $r \geq 0$, we use $C^{r}(\Omega)$ to denote the linear space of those functions $f$ on $\Omega$ for which $D^{\alpha} f \in C(\Omega)$ for all $|\alpha| \leq r$. Let $C^{\infty}(\Omega):=\bigcap_{r=0}^{\infty} C^{r}(\Omega)$ and $C_{c}^{\infty}(\Omega):=C_{c}(\Omega) \cap C^{\infty}(\Omega)$. For $k \in \mathbb{N}_{0}$ and $1 \leq p \leq \infty$, the Sobolev space $W_{p}^{k}(\Omega)$ consists of all functions $f \in L_{p}(\Omega)$ such that for each multi-index $\alpha$ with $|\alpha| \leq k, D^{\alpha} f$ exists in the distributional sense and belongs to $L_{p}(\Omega)$.

The Fourier transform of a function $f \in L_{1}\left(\mathbb{R}^{s}\right)$ is defined by

$$
\hat{f}(\xi):=\int_{\mathbb{R}^{s}} f(x) e^{-i x \cdot \xi} d x, \quad \xi \in \mathbb{R}^{s},
$$

where $i$ denotes the imaginary unit. The Fourier transform can be naturally extended to functions in $L_{2}\left(\mathbb{R}^{s}\right)$. By the Plancherel theorem, we have $\|\hat{f}\|_{2}=$ $(2 \pi)^{s / 2}\|f\|_{2}$.

For $\mu \geq 0$, we denote by $H^{\mu}\left(\mathbb{R}^{s}\right)$ the Sobolev space of all functions $f \in L_{2}\left(\mathbb{R}^{s}\right)$ such that the semi-norm

$$
|f|_{H^{\mu}\left(\mathbb{R}^{s}\right)}:=\left(\frac{1}{(2 \pi)^{s}} \int_{\mathbb{R}^{s}}|\hat{f}(\xi)|^{2}\left(\xi_{1}^{2}+\cdots+\xi_{s}^{2}\right)^{\mu} d \xi\right)^{1 / 2}
$$

is finite. The space $H^{\mu}\left(\mathbb{R}^{s}\right)$ is a Hilbert space with the inner product given by

$$
\langle f, g\rangle_{H^{\mu}\left(\mathbb{R}^{s}\right)}:=\frac{1}{(2 \pi)^{s}} \int_{\mathbb{R}^{s}} \hat{f}(\xi) \overline{\hat{g}(\xi)}\left[1+\left(\xi_{1}^{2}+\cdots+\xi_{s}^{2}\right)^{\mu}\right] d \xi, \quad f, g \in H^{\mu}\left(\mathbb{R}^{s}\right) .
$$


For $\mu>0$, the norm in $H^{\mu}\left(\mathbb{R}^{s}\right)$ is given by $\|f\|_{H^{\mu}\left(\mathbb{R}^{s}\right)}=\left(\langle f, f\rangle_{H^{\mu}\left(\mathbb{R}^{s}\right)}\right)^{1 / 2}$. For a (nonempty) open subset $\Omega$ of $\mathbb{R}^{s}$, we use $H_{0}^{\mu}(\Omega)$ to denote the closure of $C_{c}^{\infty}(\Omega)$ in $H^{\mu}\left(\mathbb{R}^{s}\right)$.

If $\mu=k \in \mathbb{N}$, then $H^{k}\left(\mathbb{R}^{s}\right)=W_{2}^{k}\left(\mathbb{R}^{s}\right)$. Suppose $u \in H^{k}\left(\mathbb{R}^{s}\right)$ for some $k \in \mathbb{N}$. For $|\alpha| \leq k$ we have $D^{\alpha} u \in L_{2}\left(\mathbb{R}^{s}\right)$ and

$$
\left(D^{\alpha} u\right)^{\wedge}(\xi)=(i \xi)^{\alpha} \hat{u}(\xi), \quad \xi \in \mathbb{R}^{s} .
$$

Let $\Delta:=D_{1}^{2}+\cdots+D_{s}^{2}$ be the Laplace operator. For $u \in H^{2}\left(\mathbb{R}^{s}\right)$ we have

$$
(\Delta u)^{\wedge}(\xi)=-\left(\xi_{1}^{2}+\cdots+\xi_{s}^{2}\right) \hat{u}(\xi), \quad \xi=\left(\xi_{1}, \ldots, \xi_{s}\right) \in \mathbb{R}^{s} .
$$

Suppose $u \in H_{0}^{2}(\Omega)$. By the Plancherel theorem we have

$$
\|\Delta u\|_{L_{2}(\Omega)}^{2}=(2 \pi)^{-s} \int_{\mathbb{R}^{s}}\left(\xi_{1}^{2}+\cdots+\xi_{s}^{2}\right)^{2}|\hat{u}(\xi)|^{2} d \xi, \quad u \in H_{0}^{2}(\Omega) .
$$

This together with (1.1) gives

$$
\|\Delta u\|_{L_{2}(\Omega)}=|u|_{H_{0}^{2}(\Omega)} \quad \forall u \in H_{0}^{2}(\Omega) .
$$

If, in addition, $\Omega$ is bounded, then we have the Poincaré inequality (see, e.g., [12, Chap. 5]):

$$
\|u\|_{H_{0}^{2}(\Omega)} \leq C|u|_{H_{0}^{2}(\Omega)} \quad \forall u \in H_{0}^{2}(\Omega),
$$

where $C$ is a constant independent of $u$. But $C$ depends on $\Omega$.

Now suppose that $\Omega$ is a bounded and connected open subset of $\mathbb{R}^{s}$, and its boundary $\partial \Omega$ is Lipschitz continuous. We are interested in the biharmonic equation with the homogeneous boundary conditions:

$$
\begin{cases}\Delta^{2} u=f & \text { in } \Omega \\ u=\frac{\partial u}{\partial n}=0 & \text { on } \partial \Omega\end{cases}
$$

where $f$ is a given function in $L_{2}(\Omega)$ and $\frac{\partial u}{\partial n}$ denotes the derivative of $u$ in the direction normal to the boundary $\partial \Omega$. For $u, v \in H_{0}^{2}(\Omega)$, it follows from (1.2) and (1.3) that

$$
\langle\Delta u, \Delta u\rangle=\|\Delta u\|_{L_{2}(\Omega)}^{2}=|u|_{H_{0}^{2}(\Omega)}^{2} \geq\|u\|_{H_{0}^{2}(\Omega)}^{2} / C .
$$

Moreover, by (1.2) we have

$$
|\langle\Delta u, \Delta v\rangle| \leq\|\Delta u\|_{L_{2}(\Omega)}\|\Delta v\|_{L_{2}(\Omega)}=|u|_{H_{0}^{2}(\Omega)}|v|_{H_{0}^{2}(\Omega)} .
$$

Hence, by the Lax-Milgram theorem, there exists a unique element $u \in H_{0}^{2}(\Omega)$ such that

$$
\langle\Delta u, \Delta v\rangle=\langle f, v\rangle \quad \forall v \in H_{0}^{2}(\Omega) .
$$

This $u$ is the weak solution to the biharmonic equation (1.4).

In order to solve the variational problem (1.5), we use finite dimensional subspaces to approximate $H_{0}^{2}(\Omega)$. Let $V$ be a subspace of $H_{0}^{2}(\Omega)$ with $\left\{v_{1}, \ldots, v_{m}\right\}$ as its basis. We look for $y_{1}, \ldots, y_{m} \in \mathbb{C}$ such that $u:=\sum_{k=1}^{m} y_{k} v_{k}$ satisfies the system of equations

$$
\left\langle\Delta u, \Delta v_{j}\right\rangle=\left\langle f, v_{j}\right\rangle, \quad j=1, \ldots, m .
$$

This system of equations can be written as

$$
\sum_{k=1}^{m} a_{j k} y_{k}=b_{j}, \quad j=1, \ldots, m
$$


where $b_{j}:=\left\langle f, v_{j}\right\rangle$ and $a_{j k}:=\left\langle\Delta v_{k}, \Delta v_{j}\right\rangle, j, k \in\{1, \ldots, m\}$. Often $v_{1}, \ldots, v_{m}$ are chosen to be finite elements over a triangulation of $\Omega$ with mesh size $h>$ 0 . Under suitable conditions, the condition number $\kappa(A)$ of the stiffness matrix $A:=\left(a_{j k}\right)_{1 \leq j, k \leq m}$ is estimated by $\kappa(A)=O\left(h^{-4}\right)$. Consequently, $A$ becomes ill-conditioned when $h$ is small. Thus, without preconditioning, it would be very difficult to solve the system of linear equations in (1.6) efficiently.

The above discussion leads us to study Riesz bases in Hilbert spaces. Let $H$ be a Hilbert space. The inner product of two elements $u$ and $v$ in $H$ is denoted by $\langle u, v\rangle$. The norm of an element $u$ in $H$ is given by $\|u\|:=\sqrt{\langle u, u\rangle}$. Let $J$ be a countable index set. A sequence $\left(v_{j}\right)_{j \in J}$ in a Hilbert space $H$ is said to be a Bessel sequence if there exists a constant $C$ such that the inequality

$$
\left\|\sum_{j \in J} c_{j} v_{j}\right\| \leq C\left(\sum_{j \in J}\left|c_{j}\right|^{2}\right)^{1 / 2}
$$

holds for every sequence $\left(c_{j}\right)_{j \in J}$ with only finitely many nonzero terms. A sequence $\left(v_{j}\right)_{j \in J}$ in $H$ is said to be a Riesz sequence if there exist two positive constants $C_{1}$ and $C_{2}$ such that the inequalities

$$
C_{1}\left(\sum_{j \in J}\left|c_{j}\right|^{2}\right)^{1 / 2} \leq\left\|\sum_{j \in J} c_{j} v_{j}\right\| \leq C_{2}\left(\sum_{j \in J}\left|c_{j}\right|^{2}\right)^{1 / 2}
$$

hold for every sequence $\left(c_{j}\right)_{j \in J}$ with only finitely many nonzero terms. We call $C_{1}$ a Riesz lower bound and $C_{2}$ a Riesz upper bound. If $\left(v_{j}\right)_{j \in J}$ is a Riesz sequence in $H$ and the linear span of $\left(v_{j}\right)_{j \in J}$ is dense in $H$, then $\left(v_{j}\right)_{j \in J}$ is said to be a Riesz basis of $H$. Let $A$ denote the matrix $\left(\left\langle v_{j}, v_{k}\right\rangle\right)_{j, k \in J}$. If (1.8) is valid, then the condition number of the matrix $A$ is no bigger than $C_{2}^{2} / C_{1}^{2}$. See [34, Chap. 4] for details.

Let us consider a model problem of the biharmonic equation (1.4) when

$$
\Omega=(0,1)^{2}:=\left\{\left(x_{1}, x_{2}\right) \in \mathbb{R}^{2}: 0<x_{1}<1,0<x_{2}<1\right\} .
$$

In this case, we first formulate a nested sequence of finite dimensional subspaces $\left(V_{n}\right)_{n \geq 3}$ of $H_{0}^{2}(\Omega)$ such that $V_{n} \subset V_{n+1}$ for all $n \geq 3$ and $\bigcup_{n=3}^{\infty} V_{n}$ is dense in $H_{0}^{2}(\Omega)$. Then we construct a suitable wavelet space $W_{n}$ such that $V_{n+1}$ is the direct sum of $V_{n}$ and $W_{n}$ for $n \geq 3$. Choose a Riesz basis for $V_{3}$ and, for each $n \geq 3$, choose a Riesz basis for $W_{n}$. For $n \geq 3$, let $\Psi_{n}$ be the union of the Riesz bases of $V_{3}$, $W_{3}, \ldots, W_{n-1}$. Under certain conditions we can prove that the condition number of the matrix $B_{n}:=(\langle\Delta u, \Delta v\rangle)_{u, v \in \Psi_{n}}$ is uniformly bounded (independent of $n$ ). Therefore, appropriately chosen Riesz bases of wavelets will give rise to efficient algorithms for numerical solutions of the biharmonic equation (1.4). In fact, more is true. We will demonstrate that the wavelet bases constructed in this paper are also good for numerical solutions of general elliptic equations of fourth-order.

Spline wavelets will be used in this paper. In [8] Chui and Wang initiated the study of semi-orthogonal wavelets generated from cardinal splines. Biorthogonal spline wavelets were constructed by Dahmen, Kunoth and Urban in [9. Many applications require wavelets with very short support. Spline wavelets with short support were investigated by Jia, Wang, and Zhou in [22, and by Han and Shen in [14. Using Hermite cubic splines, Jia and Liu in [20] constructed wavelet bases on the interval $[0,1]$ and applied those wavelets to numerical solutions to the Sturm-Liouville equation with the Dirichelet boundary condition. Spline wavelets on the interval 
$[0,1]$ with homogeneous boundary conditions were constructed in 18 . Riesz bases of multiple wavelets were studied by Han and Jiang in [13, and by Li and Xian in [25.

For polygonal domains Riesz bases of spline wavelets were constructed by Davydov and Stevenson [10] on quadrangulation, and by Jia and Liu [21] on arbitrary triangulations. However, numerical schemes based on these wavelet bases have yet to be implemented.

Here is an outline of the paper. In Section 2, we investigate cubic splines on the interval $[0,1]$ and the square $[0,1]^{2}$ with homogeneous boundary conditions. The approximation properties of these cubic splines are established and applied to convergence analysis of the finite element method for the biharmonic equation in Section 3. In order to construct wavelet bases from these cubic splines, we discuss norm equivalence induced by multilevel decompositions in Section 4, and develop a fairly general theory for Riesz bases of Hilbert spaces equipped with the induced norms in Section 5. Under the guidance of the general theory, we are able to construct wavelet bases for Sobolev spaces on the interval $(0,1)$ and the square $(0,1)^{2}$ in Section 6. In Section 7 we describe the general principle for the wavelet method and show that the condition numbers of the stiffness matrices associated with the wavelet bases are relatively small and uniformly bounded. In Sections 8 and 9 we give numerical examples to demonstrate that the algorithms based on our wavelet bases are very efficient. Finally, in Section 10, we extend our study to general elliptic equations of fourth-order and indicate that our numerical schemes also have superb performance in the general case.

\section{Approximation by CUbiC SPlines}

In this section, we investigate cubic splines on the interval $[0,1]$ and the square $[0,1]^{2}$ with homogeneous boundary conditions.

A spline is a piecewise polynomial. Let us give a definition of B-splines according to the book [3] of de Boor. Suppose $k \in \mathbb{N}$. Let $\mathbf{t}=\left(t_{j}\right)_{j \in \mathbb{Z}}$ be a sequence of real numbers such that $t_{j}<t_{j+k}$ for all $j \in \mathbb{Z}$. The B-splines of order $k$ for $\mathbf{t}$ are given by

$$
B_{j, k, \mathbf{t}}(x):=\left(t_{j+k}-t_{j}\right)\left[t_{j}, \ldots, t_{j+k}\right](\cdot-x)_{+}^{k-1}, \quad x \in \mathbb{R},
$$

where $\left[t_{j}, \ldots, t_{j+k}\right]$ denotes the $k$ th order divided difference at the points $t_{j}, \ldots, t_{j+k}$, and $a_{+}:=\max (a, 0)$. It is easily seen that each $B_{j, k, \mathbf{t}}$ is supported on $\left[t_{j}, t_{j+k}\right]$ and agrees with a polynomial of degree at most $k-1$ on each interval $\left(t_{r}, t_{r+1}\right)$ as long as $t_{r+1}>t_{r}$. Many useful properties of B-splines can be established by using the local linear functional introduced by de Boor and Fix in [4. Among other things, a polynomial $p$ of degree at most $k-1$ can be represented as a B-spline series. In other words, there exist complex numbers $c_{j}(j \in \mathbb{Z})$ such that $p=\sum_{j \in \mathbb{Z}} c_{j} B_{j, k, \mathbf{t}}$. Moreover, the B-splines $B_{j, k, \mathbf{t}}$ are locally linearly independent. To be more precise, let $(a, b)$ be a finite interval. Then only finitely many of B-splines $B_{j, k, \mathbf{t}}$ are not identically zero on $(a, b)$ and these B-splines are linearly independent on $(a, b)$.

Now suppose $k=4, N \geq 8$, and $\mathbf{t}$ is given by

$$
t_{j}= \begin{cases}j-1 & \text { for } j \leq 1, \\ j-2 & \text { for } 2 \leq j \leq N+2, \\ j-3 & \text { for } j \geq N+3\end{cases}
$$


Let $\phi_{j}:=B_{j, 4, \mathbf{t}}, j \in \mathbb{Z}$. It is easily seen that $\phi_{j}(x)=\phi_{N-j}(N-x)$ for $x \in \mathbb{R}$. Each $\phi_{j}$ is a cubic spline. Write $\phi$ for $\phi_{2}$. Then $\phi$ is supported on $[0,4]$ and

$$
\phi(x)= \begin{cases}\frac{1}{6} x^{3} & \text { for } 0 \leq x \leq 1 \\ -\frac{1}{2} x^{3}+2 x^{2}-2 x+\frac{2}{3} & \text { for } 1 \leq x \leq 2, \\ \frac{1}{2} x^{3}-4 x^{2}+10 x-\frac{22}{3} & \text { for } 2 \leq x \leq 3 \\ -\frac{1}{6} x^{3}+2 x^{2}-8 x+\frac{32}{3} & \text { for } 3 \leq x \leq 4 .\end{cases}
$$

It is easily seen that $\phi(1)=1 / 6, \phi(2)=2 / 3, \phi(3)=1 / 6$. Moreover,

$$
\phi(x)=\frac{1}{8}[\phi(2 x)+4 \phi(2 x-1)+6 \phi(2 x-2)+4 \phi(2 x-3)+\phi(2 x-4)], \quad x \in \mathbb{R} .
$$

Write $\phi_{b}$ for $\phi_{1}$. Then $\phi_{b}$ is given by

$$
\phi_{b}(x)= \begin{cases}\frac{1}{12}\left(-11 x^{3}+18 x^{2}\right) & \text { for } 0 \leq x \leq 1, \\ \frac{1}{12}\left(7 x^{3}-36 x^{2}+54 x-18\right) & \text { for } 1 \leq x \leq 2, \\ \frac{1}{6}(3-x)^{3} & \text { for } 2 \leq x \leq 3, \\ 0 & \text { for } x \in \mathbb{R} \backslash[0,3] .\end{cases}
$$

It is easily seen that $\phi_{b}(1)=7 / 12$ and $\phi_{b}(2)=1 / 6$. Moreover,

$$
\phi_{b}(x)=\frac{1}{4} \phi_{b}(2 x)+\frac{11}{16} \phi(2 x)+\frac{1}{2} \phi(2 x-1)+\frac{1}{8} \phi(2 x-2), \quad x \in \mathbb{R} .
$$

We have $\phi_{b}^{\prime \prime} \in L_{2}(\mathbb{R})$ and

$$
\phi_{b}^{\prime \prime}(x)= \begin{cases}\frac{1}{2}(-11 x+6) & \text { for } 0<x \leq 1 \\ \frac{1}{2}(7 x-12) & \text { for } 1<x \leq 2 \\ 3-x & \text { for } 2<x \leq 3 \\ 0 & \text { for } x \in \mathbb{R} \backslash[0,3] .\end{cases}
$$

It follows that $\lim _{x \rightarrow 0-} \phi_{b}^{\prime \prime}(x)=0$ and $\lim _{x \rightarrow 0+} \phi_{b}^{\prime \prime}(x)=3$. Hence, $\phi_{b}^{\prime \prime \prime}=g+3 \delta$, where $g$ is a piecewise constant function supported on $[0,3]$ and $\delta$ is the Dirac delta function. By taking the Fourier transform of both sides of the equation $\phi_{b}^{\prime \prime \prime}=g+3 \delta$ we obtain $(i \xi)^{3} \hat{\phi}_{b}(\xi)=\hat{g}(\xi)+3$ for $\xi \in \mathbb{R}$. Consequently, for $0<\mu<5 / 2$ we have

$$
\int_{|\xi|>1}|\xi|^{2 \mu}\left|\hat{\phi}_{b}(\xi)\right|^{2} d \xi=\int_{|\xi|>1} \frac{|\hat{g}(\xi)+3|^{2}}{|\xi|^{2(3-\mu)}} d \xi<\infty .
$$

Moreover,

$$
\int_{|\xi| \leq 1}|\xi|^{2 \mu}\left|\hat{\phi}_{b}(\xi)\right|^{2} d \xi \leq \int_{|\xi| \leq 1}\left|\hat{\phi}_{b}(\xi)\right|^{2} d \xi<\infty .
$$

This shows that $\phi_{b} \in H^{\mu}(\mathbb{R})$ for $0<\mu<5 / 2$. An analogous argument shows that $\phi \in H^{\mu}(\mathbb{R})$ for $0<\mu<7 / 2$.

We see that $\phi$ is supported on $[0,4]$. Consequently, $\phi(\cdot-k)$ vanishes on $(1,2)$ for $k \leq-3$ or $k \geq 2$. Moreover, the B-splines $\phi(\cdot-k)(k=-2,-1,0,1)$ are linearly independent on $(1,2)$. Hence, there exists a continuous function $\tilde{\varphi}$ supported on $[1,2]$ such that

$$
\langle\tilde{\varphi}, \phi(\cdot-k)\rangle=\delta_{0 k} \quad \forall k \in \mathbb{Z},
$$


where $\delta_{j k}$ denotes the Kronecker symbol: $\delta_{j k}=1$ for $j=k$ and $\delta_{j k}=0$ for $j \neq k$. We observe that

$$
\phi_{k}= \begin{cases}\phi(\cdot-k+1) & \text { for } k \leq-3, \\ \phi(\cdot-k+2) & \text { for } k=2, \ldots, N-2, \\ \phi(\cdot-k+3) & \text { for } k \geq N+3\end{cases}
$$

Accordingly, we define $\tilde{\phi}_{j}$ for $j \in \mathbb{Z} \backslash([-3,3] \cup[N-2, N+4])$ as follows:

$$
\tilde{\phi}_{j}:= \begin{cases}\tilde{\varphi}(\cdot-j+1) & \text { for } j \leq-4, \\ \tilde{\varphi}(\cdot-j+2) & \text { for } j=4, \ldots, N-3, \\ \tilde{\varphi}(\cdot-j+3) & \text { for } j \geq N+5 .\end{cases}
$$

It is easily verified that $\left\langle\tilde{\phi}_{j}, \phi_{k}\right\rangle=\delta_{j k}$ for all $j \in \mathbb{Z} \backslash([-3,3] \cup[N-2, N+4])$ and $k \in \mathbb{Z}$. Suppose $-3 \leq j \leq 3$. By the local linear independence of the Bsplines $\phi_{k}(k \in \mathbb{Z})$, we can find a continuous function $\tilde{\phi}_{j}$ supported on $[j-1, j]$ such that $\left\langle\tilde{\phi}_{j}, \phi_{k}\right\rangle=\delta_{j k}$ for all $k \in \mathbb{Z}$. Similarly, for $N-2 \leq j \leq N+4$ we can find a continuous function $\tilde{\phi}_{j}$ supported on $[j, j+1]$ such that $\left\langle\tilde{\phi}_{j}, \phi_{k}\right\rangle=\delta_{j k}$ for all $k \in \mathbb{Z}$. We conclude that $\left\langle\tilde{\phi}_{j}, \phi_{k}\right\rangle=\delta_{j k}$ for all $j \in \mathbb{Z}$ and $k \in \mathbb{Z}$. In light of our construction, there is a constant $M$ independent of $N$ such that $\left\|\tilde{\phi}_{j}\right\|_{\infty} \leq M$ for all $j \in \mathbb{Z}$. Consequently, $\left(\phi_{j}\right)_{j \in \mathbb{Z}}$ is a Riesz sequence in $L_{2}(\mathbb{R})$.

Let $Q$ be the linear operator given by

$$
Q u:=\sum_{j \in \mathbb{Z}}\left\langle u, \tilde{\phi}_{j}\right\rangle \phi_{j},
$$

where $u$ is a locally integrable function on $\mathbb{R}$. In particular, $Q u$ is well defined for $u$ in $L_{2}(\mathbb{R})$. Since $\left\|\tilde{\phi}_{j}\right\|_{\infty} \leq M$ for all $j \in \mathbb{Z}, Q$ is a bounded operator on $L_{2}(\mathbb{R})$. Moreover, if $u$ is supported on $[0, N]$, then $\left\langle u, \tilde{\phi}_{j}\right\rangle=0$ for $j \leq 0$ or $j \geq N$. But $\phi_{j}$ is supported in $[0, N]$ for $1 \leq j \leq N-1$. Therefore, $Q u$ is supported on $[0, N]$, provided $u$ is supported on $[0, N]$. We have $Q \phi_{j}=\phi_{j}$ for all $j \in \mathbb{Z}$. A polynomial $p \in \Pi_{3}$ can be represented as $p=\sum_{j \in \mathbb{Z}} c_{j} \phi_{j}$. Hence, $Q p=p$ for all $p \in \Pi_{3}$.

Now choose $N=2^{n}$ for some $n \geq 3$. For $j \in \mathbb{Z}$, let $\phi_{n, j}(x):=2^{n / 2} \phi_{j}\left(2^{n} x\right)$ and $\tilde{\phi}_{n, j}(x):=2^{n / 2} \tilde{\phi}_{j}\left(2^{n} x\right), x \in \mathbb{R}$. Then $\left\langle\tilde{\phi}_{n, j}, \phi_{n, k}\right\rangle=\delta_{j k}$ for all $j, k \in \mathbb{Z}$. Let $Q_{n}$ be the linear operator given by

$$
Q_{n} u=\sum_{j \in \mathbb{Z}}\left\langle u, \tilde{\phi}_{n, j}\right\rangle \phi_{n, j},
$$

where $u$ is a locally integrable function on $\mathbb{R}$. Clearly, $Q_{n} \phi_{n, j}=\phi_{n, j}$ for all $j \in \mathbb{Z}$. By $\left\|Q_{n}\right\|$ we denote the norm of $Q_{n}$ as an operator on $L_{2}(\mathbb{R})$. Then $\left\|Q_{n}\right\|=\|Q\|$ for all $n \geq 3$. Since $Q_{n} p=p$ for all $p \in \Pi_{3}$, for $0 \leq m \leq 3$ we have (see [16] and [19])

$$
\left\|Q_{n} u-u\right\|_{H^{m}(\mathbb{R})} \leq C\left(1 / 2^{n}\right)^{4-m}|u|_{H^{4}(\mathbb{R})}, \quad u \in H^{4}(\mathbb{R}),
$$

where $C$ is a constant independent of $n$ and $u$.

For $n \geq 3$ and $j=1, \ldots, 2^{n}-1, \phi_{n, j}$ and $\tilde{\phi}_{n, j}$ are supported in the interval $[0,1]$. Let $V_{n}(0,1)$ be the linear span of $\left\{\phi_{n, j}: j=1, \ldots, 2^{n}-1\right\}$. Since $\phi_{n, j} \in H^{\mu}(\mathbb{R})$ for $0<\mu<5 / 2, V_{n}(0,1)$ is a closed subspace of $H_{0}^{\mu}(0,1)$ for $0<\mu<5 / 2$ and its dimension is $2^{n}-1$. Moreover, $V_{n}(0,1) \subset V_{n+1}(0,1)$ for $n \geq 3$. Recall that $L_{2}(0,1)$ is regarded as the subspace of $L_{2}(\mathbb{R})$ consisting of all functions in $L_{2}(\mathbb{R})$ that vanish outside $(0,1)$. It is easily seen that $\left\{\phi_{n, j}: j=1, \ldots, 2^{n}-1\right\}$ is a Riesz sequence in 
$L_{2}(0,1)$ with Riesz bounds independent of $n$. If $u \in L_{2}(0,1)$, then $\left\langle u, \tilde{\phi}_{n, j}\right\rangle=0$ for $j \notin\left\{1, \ldots, 2^{n}-1\right\}$. Hence, for $n \geq 3, Q_{n}$ maps $L_{2}(0,1)$ onto $V_{n}(0,1)$.

For $n \geq 3$ and $j=\left(j_{1}, j_{2}\right) \in \mathbb{Z}^{2}$, let

$$
\begin{aligned}
\phi_{n, j}(x)=\phi_{n, j_{1}}\left(x_{1}\right) \phi_{n, j_{2}}\left(x_{2}\right) \quad \text { and } \quad \tilde{\phi}_{n, j}(x)=\tilde{\phi}_{n, j_{1}}\left(x_{1}\right) \tilde{\phi}_{n, j_{2}}\left(x_{2}\right), & \\
x & =\left(x_{1}, x_{2}\right) \in \mathbb{R}^{2} .
\end{aligned}
$$

Then $\left\langle\tilde{\phi}_{n, j}, \phi_{n, k}\right\rangle=\delta_{j k}$ for all $j, k \in \mathbb{Z}^{2}$. Let $\mathcal{Q}_{n}$ be the linear operator given by

$$
\mathcal{Q}_{n} u=\sum_{j \in \mathbb{Z}^{2}}\left\langle u, \tilde{\phi}_{n, j}\right\rangle \phi_{n, j}
$$

where $u$ is a locally integrable function on $\mathbb{R}^{2}$. Clearly, $\mathcal{Q}_{n} \phi_{n, j}=\phi_{n, j}$ for all $j \in \mathbb{Z}^{2}$. Consequently, $\mathcal{Q}_{n} p=p$ for every bivariate polynomial $p$ of degree at most 3 . Hence, for $0 \leq m \leq 3$, there exists a constant $C$ independent of $n$ and $u$ such that

$$
\left\|u-\mathcal{Q}_{n} u\right\|_{H^{m}\left(\mathbb{R}^{2}\right)} \leq C\left(1 / 2^{n}\right)^{4-m}|u|_{H^{4}\left(\mathbb{R}^{2}\right)}, \quad u \in H^{4}\left(\mathbb{R}^{2}\right) .
$$

Suppose $v_{1}, v_{2} \in H^{\mu}(\mathbb{R})$ for some $\mu>0$. Let $v$ be the function on $\mathbb{R}^{2}$ given by $v\left(x_{1}, x_{2}\right):=v_{1}\left(x_{1}\right) v_{2}\left(x_{2}\right),\left(x_{1}, x_{2}\right) \in \mathbb{R}^{2}$. Then $\hat{v}\left(\xi_{1}, \xi_{2}\right)=\hat{v}_{1}\left(\xi_{1}\right) \hat{v}_{2}\left(\xi_{2}\right)$ for $\left(\xi_{1}, \xi_{2}\right) \in \mathbb{R}^{2}$. Consequently,

$$
\int_{\mathbb{R}^{2}}\left|\xi_{1}\right|^{2 \mu}\left|\hat{v}\left(\xi_{1}, \xi_{2}\right)\right|^{2} d \xi_{1} d \xi_{2}=\int_{\mathbb{R}}\left|\xi_{1}\right|^{2 \mu}\left|\hat{v}_{1}\left(\xi_{1}\right)\right|^{2} d \xi_{1} \int_{\mathbb{R}}\left|\hat{v}_{2}\left(\xi_{2}\right)\right|^{2} d \xi_{2}<\infty
$$

and

$$
\int_{\mathbb{R}^{2}}\left|\xi_{2}\right|^{2 \mu}\left|\hat{v}\left(\xi_{1}, \xi_{2}\right)\right|^{2} d \xi_{1} d \xi_{2}=\int_{\mathbb{R}}\left|\hat{v}_{1}\left(\xi_{1}\right)\right|^{2} d \xi_{1} \int_{\mathbb{R}}\left|\xi_{2}\right|^{2 \mu}\left|\hat{v}_{2}\left(\xi_{2}\right)\right|^{2} d \xi_{2}<\infty .
$$

This shows $v \in H^{\mu}\left(\mathbb{R}^{2}\right)$. For $j=\left(j_{1}, j_{2}\right) \in \mathbb{Z}^{2}$, we have $\phi_{n, j_{1}} \in H^{\mu}(\mathbb{R})$ and $\phi_{n, j_{2}} \in H^{\mu}(\mathbb{R})$ for $0<\mu<5 / 2$. Hence, $\phi_{n, j} \in H^{\mu}\left(\mathbb{R}^{2}\right)$ for $0<\mu<5 / 2$.

Let $J_{n}:=\left\{\left(j_{1}, j_{2}\right) \in \mathbb{Z}^{2}: 1 \leq j_{1}, j_{2} \leq 2^{n}-1\right\}$. For $n \geq 3$ and $j \in J_{n}, \phi_{n, j}$ and $\tilde{\phi}_{n, j}$ are supported in the square $[0,1]^{2}$. Let $V_{n}\left((0,1)^{2}\right)$ be the linear span of $\left\{\phi_{n, j}: j \in J_{n}\right\}$. Then $V_{n}\left((0,1)^{2}\right)$ is a closed subspace of $H_{0}^{\mu}\left((0,1)^{2}\right)$ for $0<\mu<5 / 2$ and its dimension is $\left(2^{n}-1\right)^{2}$. Moreover, $V_{n}\left((0,1)^{2}\right) \subset V_{n+1}\left((0,1)^{2}\right)$. Recall that $L_{2}\left((0,1)^{2}\right)$ is regarded as the subspace of $L_{2}\left(\mathbb{R}^{2}\right)$ consisting of all functions in $L_{2}\left(\mathbb{R}^{2}\right)$ that vanish outside $(0,1)^{2}$. It is easily seen that $\left\{\phi_{n, j}: j \in J_{n}\right\}$ is a Riesz sequence in $L_{2}\left((0,1)^{2}\right)$ with Riesz bounds independent of $n$. If $u \in L_{2}\left((0,1)^{2}\right)$, then $\left\langle u, \tilde{\phi}_{n, j}\right\rangle=0$ for $n \geq 3$ and $j \notin J_{n}$. Hence, for $n \geq 3, \mathcal{Q}_{n}$ maps $L_{2}\left((0,1)^{2}\right)$ onto $V_{n}\left((0,1)^{2}\right)$.

\section{Convergence Rates}

Throughout this section we assume that $\Omega$ is the unit square $(0,1)^{2}$. We also write $V_{n}$ for $V_{n}\left((0,1)^{2}\right)$. Given $f \in L_{2}(\Omega)$, let $u \in H_{0}^{2}(\Omega)$ be the unique solution to the equation (1.5). Since the inner angle at each corner of the square is $\pi / 2$, we have $u \in H^{4}(\Omega)$, by [2, Theorem 7]. Moreover, $|u|_{H^{4}(\Omega)} \leq C\|f\|_{L_{2}(\Omega)}$ for some constant $C$ independent of $f$. For $n \geq 3$, let $u_{n}$ be the unique solution in $V_{n}$ to the following equation:

$$
\left\langle\Delta u_{n}, \Delta v\right\rangle=\langle f, v\rangle \quad \forall v \in V_{n} .
$$

In this section we will prove that

$$
\left\|u_{n}-u\right\|_{H^{m}(\Omega)} \leq C\left(1 / 2^{n}\right)^{4-m}\|f\|_{L^{2}(\Omega)} \quad \forall n \geq 3,
$$


where $m=0,1,2$ and $C$ is a constant independent of $f$ and $n$. For relevant results of finite element methods, the reader is referred to the book [31] of Strang and Fix, and the book [5] of Brenner and Scott.

The function $u \in H^{4}(\Omega)$ is a weak solution to the biharmonic equation (1.4). By changing its values on a set of measure zero if necessary, $u$ becomes a function in $C^{2}(\bar{\Omega})$, in light of the Sobolev embedding theorem (see [12, Chap. 5]). Since $u \in H_{0}^{2}(\Omega)$, there exists a sequence $\left(g_{n}\right)_{n=1,2, \ldots}$ in $C_{c}^{\infty}(\Omega)$ such that $\lim _{n \rightarrow \infty} \| g_{n}-$ $u \|_{H^{2}(\Omega)}=0$. By the embedding theorem, $\left\|g_{n}-u\right\|_{C(\bar{\Omega})} \leq C\left\|g_{n}-u\right\|_{H^{2}(\Omega)}$, where $C$ is a constant independent of $n$. Hence, $\lim _{n \rightarrow \infty}\left\|g_{n}-u\right\|_{C(\bar{\Omega})}=0$. But $g_{n}(x)=0$ for all $n \in \mathbb{N}$ and $x \in \partial \Omega$. Consequently, $u(x)=0$ for all $x \in \partial \Omega$. Let $y$ be a nonzero vector in $\mathbb{R}^{2}$. Then $\lim _{n \rightarrow \infty}\left\|D_{y} g_{n}-D_{y} u\right\|_{H^{1}(\Omega)}=0$. Let $\gamma$ be a line segment $\subset \partial \Omega$ that does not contain any of the four corners of $\Omega$. By the trace theorem (see [12, p. 258]), we have

$$
\left\|D_{y} g_{n}-D_{y} u\right\|_{L_{2}(\gamma)} \leq C\left\|D_{y} g_{n}-D_{y} u\right\|_{H^{1}(\Omega)},
$$

where $C$ is a constant independent of $n$. It follows that $\lim _{n \rightarrow \infty}\left\|D_{y} g_{n}-D_{y} u\right\|_{L_{2}(\gamma)}$ $=0$. But $D_{y} g_{n}=0$ on $\gamma$ for every $n \in \mathbb{N}$. Therefore, $\left\|D_{y} u\right\|_{L_{2}(\gamma)}=0$. Note that $D_{y} u$ is continuous on $\partial \Omega$. This shows that $D_{y} u(x)=0$ for all $x \in \gamma$. Consequently, $D_{y} u(x)=0$ for all $x \in \partial \Omega$. In particular, $D_{1} u(x)=D_{2} u(x)=0$ for all $x \in \partial \Omega$.

In order to prove (3.2), we first establish the following result on approximation by cubic splines:

$$
\begin{aligned}
& \inf _{v \in V_{n}}\|u-v\|_{H^{m}(\Omega)} \leq C\left(1 / 2^{n}\right)^{4-m}|u|_{H^{4}(\Omega)}, \\
& \text { for } n \geq 3 \text { and } u \in H_{0}^{2}(\Omega) \cap H^{4}(\Omega),
\end{aligned}
$$

where $0 \leq m \leq 3$ and $C$ is a constant independent of $n$ and $u$. If we extend $u$ to $\mathbb{R}^{2}$ by setting $u(x)=0$ for $x \in \mathbb{R}^{2} \backslash \Omega$, then the extended function will be in $H^{2}\left(\mathbb{R}^{2}\right)$ but, in general, will not be in $H^{4}\left(\mathbb{R}^{2}\right)$. Thus, (2.4) cannot be applied directly to the current situation.

For $N \geq 8$, let $\mathbf{t}:=\left(t_{j}\right)_{-1 \leq j \leq N+5}$ be the knot sequence given by

$$
t_{j}= \begin{cases}0 & \text { for }-1 \leq j \leq 2, \\ j-2 & \text { for } 3 \leq j \leq N+1, \\ N & \text { for } N+2 \leq j \leq N+5 .\end{cases}
$$

For $j \in\{-1,0, N, N+1\}$, let $\phi_{j}$ be the B-spline $B_{j, 4, \mathbf{t}}$. For $1 \leq j \leq N-1, \phi_{j}$ is the same as in $\S 2$. By using an argument analogous to that given in $\S 2$, we can find real-valued continuous functions $\tilde{\phi}_{j}(-1 \leq j \leq N+1)$ with the following properties:

(a) $\left\langle\tilde{\phi}_{j}, \phi_{k}\right\rangle=\delta_{j k}$ for all $j, k \in\{-1,0, \ldots, N, N+1\}$;

(b) $\left\|\tilde{\phi}_{j}\right\|_{\infty} \leq M$ for $-1 \leq j \leq N+1$, where $M$ is a constant independent of $N$;

(c) $\tilde{\phi}_{j}$ is supported on the interval $E_{j}$, where $E_{j}:=[0,1]$ for $j=-1,0, E_{j}:=$ $[j-1, j]$ for $j=1, \ldots, N-1$, and $E_{j}:=[N-1, N]$ for $j=N, N+1$.

Given $n \geq 3$, we choose $N:=2^{n}$. For $j \in\{-1,0, \ldots, N, N+1\}$, let

$$
\phi_{n, j}(x)=2^{n / 2} \phi_{j}\left(2^{n} x\right) \quad \text { and } \quad \tilde{\phi}_{n, j}(x)=2^{n / 2} \tilde{\phi}_{j}\left(2^{n} x\right), \quad x \in[0,1] .
$$

For $n \geq 3$ and $j \in \tilde{J}_{n}:=\left\{\left(j_{1}, j_{2}\right):-1 \leq j_{1}, j_{2} \leq 2^{n}+1\right\}$, let

$$
\begin{aligned}
\phi_{n, j}(x)=\phi_{n, j_{1}}\left(x_{1}\right) \phi_{n, j_{2}}\left(x_{2}\right) \text { and } \quad \tilde{\phi}_{n, j}(x)=\tilde{\phi}_{n, j_{1}}\left(x_{1}\right) \tilde{\phi}_{n, j_{2}}\left(x_{2}\right), \\
x=\left(x_{1}, x_{2}\right) \in[0,1]^{2} .
\end{aligned}
$$


Then $\left\langle\tilde{\phi}_{n, j}, \phi_{n, k}\right\rangle=\delta_{j k}$ for all $j, k \in \tilde{J}_{n}$. Let $\tilde{\mathcal{Q}}_{n}$ be the linear operator given by

$$
\tilde{\mathcal{Q}}_{n} u=\sum_{j \in \tilde{J}_{n}}\left\langle u, \tilde{\phi}_{n, j}\right\rangle \phi_{n, j},
$$

where $u$ is a locally integrable function on $[0,1]^{2}$. If $p$ is the restriction of a bivariate polynomial of degree at most 3 to $[0,1]^{2}$, then $\tilde{\mathcal{Q}}_{n} p=p$. By the results on approximation in [23] and [16, we obtain

$$
\left\|u-\tilde{\mathcal{Q}}_{n} u\right\|_{H^{m}(\Omega)} \leq C\left(1 / 2^{n}\right)^{4-m}|u|_{H^{4}(\Omega)}, \quad u \in H^{4}(\Omega),
$$

where $0 \leq m \leq 3$ and $C$ is a constant independent of $n$ and $u$.

For $n \geq 3$, let $\mathcal{Q}_{n}$ be the linear operator given in (2.3). Recall that $J_{n}$ is the set $\left\{\left(j_{1}, j_{2}\right): 1 \leq j_{1}, j_{2} \leq 2^{n}-1\right\}$. If $u$ is a locally integrable function on $[0,1]^{2}$, then $\left\langle u, \tilde{\phi}_{n, j}\right\rangle=0$ for $j \in \mathbb{Z}^{2} \backslash J_{n}$. Hence, $\mathcal{Q}_{n} u \in V_{n}$ for $n \geq 3$. Taking (3.4) into account, we see that in order to establish (3.3), it suffices to show

$$
\begin{array}{rr}
\left\|\tilde{\mathcal{Q}}_{n} u-\mathcal{Q}_{n} u\right\|_{H^{m}(\Omega)} \leq C\left(1 / 2^{n}\right)^{4-m}|u|_{H^{4}(\Omega)}, \quad u \in H^{4}(\Omega) \cap H_{0}^{2}(\Omega), \\
0 \leq m \leq 3 .
\end{array}
$$

We have

$$
\tilde{\mathcal{Q}}_{n} u-\mathcal{Q}_{n} u=\sum_{j \in \tilde{J}_{n} \backslash J_{n}}\left\langle u, \tilde{\phi}_{n, j}\right\rangle \phi_{n, j} .
$$

It follows that (see [17, §3])

$$
\left\|\tilde{\mathcal{Q}}_{n} u-\mathcal{Q}_{n} u\right\|_{H^{m}(\Omega)} \leq C\left(2^{n}\right)^{m}\left(\sum_{j \in \tilde{J}_{n} \backslash J_{n}}\left|\left\langle u, \tilde{\phi}_{n, j}\right\rangle\right|^{2}\right)^{1 / 2}, \quad 0 \leq m \leq 3 .
$$

We observe that $\left(j_{1}, j_{2}\right) \in \tilde{J}_{n} \backslash J_{n}$ if and only if $j_{1}$ or $j_{2}$ belongs to $\{-1,0, N, N+1\}$. Let us consider the case $j_{1}=0$. Other cases can be treated similarly. Set $h:=1 / 2^{n}$. Since $\tilde{\phi}_{n, 0}$ is supported on $[0, h]$, for $j=\left(j_{1}, j_{2}\right)$ with $j_{1}=0$ we have

$$
\left\langle u, \tilde{\phi}_{n, j}\right\rangle=\int_{0}^{1} \int_{0}^{h} u\left(x_{1}, x_{2}\right) \tilde{\phi}_{n, 0}\left(x_{1}\right) \tilde{\phi}_{n, j_{2}}\left(x_{2}\right) d x_{1} d x_{2} .
$$

Let $p_{1}, p_{2}, p_{3}, p_{4}$ be the unique cubic polynomials on $\mathbb{R}$ such that

$$
\left[\begin{array}{llll}
p_{1}(0) & p_{1}^{\prime}(0) & p_{1}(h) & p_{1}^{\prime}(h) \\
p_{2}(0) & p_{2}^{\prime}(0) & p_{2}(h) & p_{2}^{\prime}(h) \\
p_{3}(0) & p_{3}^{\prime}(0) & p_{3}(h) & p_{3}^{\prime}(h) \\
p_{4}(0) & p_{4}^{\prime}(0) & p_{4}(h) & p_{4}^{\prime}(h)
\end{array}\right]=\left[\begin{array}{llll}
1 & 0 & 0 & 0 \\
0 & 1 & 0 & 0 \\
0 & 0 & 1 & 0 \\
0 & 0 & 0 & 1
\end{array}\right]
$$

where $p^{\prime}$ denotes the derivative of $p$. For $u \in H^{4}(\Omega) \cap H_{0}^{2}(\Omega)$ and $x=\left(x_{1}, x_{2}\right) \in \bar{\Omega}$, let

$w\left(x_{1}, x_{2}\right):=u\left(0, x_{2}\right) p_{1}\left(x_{1}\right)+D_{1} u\left(0, x_{2}\right) p_{2}\left(x_{1}\right)+u\left(h, x_{2}\right) p_{3}\left(x_{1}\right)+D_{1} u\left(h, x_{2}\right) p_{4}\left(x_{1}\right)$. It is easily seen that $w\left(0, x_{2}\right)=u\left(0, x_{2}\right), D_{1} w\left(0, x_{2}\right)=D_{1} u\left(0, x_{2}\right), w\left(h, x_{2}\right)=$ $u\left(h, x_{2}\right)$, and $D_{1} w\left(h, x_{2}\right)=D_{1} u\left(h, x_{2}\right)$. Hence, by Theorem (4.4.4) of [],

$$
\int_{0}^{h}\left|u\left(x_{1}, x_{2}\right)-w\left(x_{1}, x_{2}\right)\right|^{2} d x_{1} \leq C_{1} h^{8} \int_{0}^{h}\left|D_{1}^{4} u\left(x_{1}, x_{2}\right)\right|^{2} d x_{1}, \quad 0 \leq x_{2} \leq 1,
$$

where $C_{1}$ is a constant independent of $u, h$, and $x_{2}$. Since $u \in H_{0}^{2}(\Omega) \cap H^{4}(\Omega)$, we have $u\left(0, x_{2}\right)=0$ and $D_{1} u\left(0, x_{2}\right)=0$ for $0 \leq x_{2} \leq 1$. Moreover, on the interval 
$(0, h), p_{3}$ and $p_{4}$ can be expressed as linear combinations of $\phi_{n, 1}$ and $\phi_{n, 2}$ (see the definition of $\phi_{1}$ and $\phi_{2}$ given in $\left.\S 2\right)$. But $\left\langle\tilde{\phi}_{n, 0}, \phi_{n, k}\right\rangle=0$ for $k=1,2$. Hence,

$$
\int_{0}^{h} w\left(x_{1}, x_{2}\right) \tilde{\phi}_{n, 0}\left(x_{1}\right) d x_{1}=0, \quad 0 \leq x_{2} \leq 1 .
$$

We conclude that

$$
\left\langle u, \tilde{\phi}_{n,\left(0, j_{2}\right)}\right\rangle=\int_{0}^{1} \int_{0}^{h}\left[u\left(x_{1}, x_{2}\right)-w\left(x_{1}, x_{2}\right)\right] \tilde{\phi}_{n, 0}\left(x_{1}\right) \tilde{\phi}_{n, j_{2}}\left(x_{2}\right) d x_{1} d x_{2} .
$$

Recall that $\tilde{\phi}_{n, j_{2}}$ is supported on the interval $E_{j_{2}}$. Let $\chi_{j_{2}}$ denote the characteristic function of $E_{j_{2}}$. We deduce from the above equality that

$$
\left\langle u, \tilde{\phi}_{n,\left(0, j_{2}\right)}\right\rangle=\int_{0}^{1} \int_{0}^{h}\left[u\left(x_{1}, x_{2}\right)-w\left(x_{1}, x_{2}\right)\right] \tilde{\phi}_{n, 0}\left(x_{1}\right) \tilde{\phi}_{n, j_{2}}\left(x_{2}\right) \chi_{j_{2}}\left(x_{2}\right) d x_{1} d x_{2} .
$$

Since $\left\|\tilde{\phi}_{n, 0}\right\|_{L_{2}(0,1)} \leq M$ and $\left\|\tilde{\phi}_{n, j_{2}}\right\|_{L_{2}(0,1)} \leq M$, by the Schwarz inequality we obtain

$$
\left|\left\langle u, \tilde{\phi}_{n,\left(0, j_{2}\right)}\right\rangle\right|^{2} \leq C_{2} \int_{0}^{1} \int_{0}^{h}\left|u\left(x_{1}, x_{2}\right)-w\left(x_{1}, x_{2}\right)\right|^{2} \chi_{j_{2}}\left(x_{2}\right) d x_{1} d x_{2} .
$$

Here and in what follows, $C_{i}(i \in \mathbb{N})$ denotes a constant independent of $u$ and $h$. It follows that

$$
\sum_{j_{2}=-1}^{N+1}\left|\left\langle u, \tilde{\phi}_{n,\left(0, j_{2}\right)}\right\rangle\right|^{2} \leq C_{2} \int_{0}^{1} \int_{0}^{h}\left|u\left(x_{1}, x_{2}\right)-w\left(x_{1}, x_{2}\right)\right|^{2} \sum_{j_{2}=-1}^{N+1} \chi_{j_{2}}\left(x_{2}\right) d x_{1} d x_{2} .
$$

But $\sum_{j_{2}=-1}^{N+1} \chi_{j_{2}}\left(x_{2}\right) \leq 4$ for $0 \leq x_{2} \leq 1$. Thus, the above inequality together with (3.7) gives

$$
\left(\sum_{j_{2}=-1}^{N+1}\left|\left\langle u, \tilde{\phi}_{n,\left(0, j_{2}\right)}\right\rangle\right|^{2}\right)^{1 / 2} \leq C_{3}\left(2^{-n}\right)^{4}|u|_{H^{4}(\Omega)}, \quad u \in H_{0}^{2}(\Omega) \cap H^{4}(\Omega) .
$$

By using the same argument as for the case $j=\left(0, j_{2}\right)$, we obtain

$$
\left(\sum_{j \in \tilde{J}_{n} \backslash J_{n}}\left|\left\langle u, \tilde{\phi}_{n, j}\right\rangle\right|^{2}\right)^{1 / 2} \leq C_{4}\left(2^{-n}\right)^{4}|u|_{H^{4}(\Omega)}, \quad u \in H_{0}^{2}(\Omega) \cap H^{4}(\Omega) .
$$

This in connection with (3.6) gives (3.5). Finally, since $\mathcal{Q}_{n} u \in V_{n}$, the desired estimate (3.3) follows from (3.4) and (3.5).

We are in a position to establish (3.2). Our proof follows the lines in $\S 5.9$ of [5].

Theorem 3.1. Given $f \in L_{2}(\Omega)$, let $u$ be the unique solution in $H_{0}^{2}(\Omega)$ to the equation (1.5). For $n \geq 3$, let $u_{n}$ be the unique solution in $V_{n}$ to the equation (3.1). Then the estimate (3.2) holds for $m=0,1,2$ and a constant $C$ independent of $f$ and $n$.

Proof. By (1.5) and (3.1) we have $\left\langle\Delta\left(u-u_{n}\right), \Delta w\right\rangle=0$ for all $w \in V_{n}$. Suppose $v \in V_{n}$. Then $u_{n}-v \in V_{n}$. Hence,

$$
\left\|\Delta\left(u-u_{n}\right)\right\|_{L_{2}(\Omega)}^{2}=\left\langle\Delta\left(u-u_{n}\right), \Delta\left(u-u_{n}\right)\right\rangle=\left\langle\Delta\left(u-u_{n}\right), \Delta(u-v)\right\rangle .
$$


Taking (1.2) into account, we obtain

$$
\begin{aligned}
\left\|\Delta\left(u-u_{n}\right)\right\|_{L_{2}(\Omega)}^{2} & \leq\left\|\Delta\left(u-u_{n}\right)\right\|_{L_{2}(\Omega)}\|\Delta(u-v)\|_{L_{2}(\Omega)} \\
& =\left|u-u_{n}\right|_{H^{2}(\Omega)}|u-v|_{H^{2}(\Omega)} \quad \forall v \in V_{n} .
\end{aligned}
$$

Write $h:=2^{-n}$. By (3.3) we have

$$
\left\|\Delta\left(u-u_{n}\right)\right\|_{L_{2}(\Omega)} \leq \inf _{v \in V_{n}}|u-v|_{H^{2}(\Omega)} \leq C h^{2}\|f\|_{L_{2}(\Omega)} .
$$

Suppose $g \in L_{2}(\Omega)$. Let $w$ be the unique element in $H_{0}^{2}(\Omega)$ such that

$$
\langle\Delta w, \Delta v\rangle=\langle g, v\rangle \quad \forall v \in H_{0}^{2}(\Omega) .
$$

Then $w \in H_{0}^{2}(\Omega) \cap H^{4}(\Omega)$ and $\Delta^{2} w=g$. Hence,

$$
\left\langle u-u_{n}, g\right\rangle=\left\langle u-u_{n}, \Delta^{2} w\right\rangle=\left\langle\Delta\left(u-u_{n}\right), \Delta w\right\rangle .
$$

Let $w_{n}$ be the unique element in $V_{n}$ such that

$$
\left\langle\Delta w_{n}, \Delta v\right\rangle=\langle g, v\rangle \quad \forall v \in V_{n} .
$$

By using the same argument as in the preceding paragraph, we have

$$
\left\|\Delta\left(w-w_{n}\right)\right\|_{L_{2}(\Omega)} \leq C h^{2}\|g\|_{L_{2}(\Omega)} .
$$

Moreover, it follows from (3.9) that

$$
\left\langle u-u_{n}, g\right\rangle=\left\langle\Delta\left(u-u_{n}\right), \Delta w\right\rangle-\left\langle\Delta\left(u-u_{n}\right), \Delta w_{n}\right\rangle=\left\langle\Delta\left(u-u_{n}\right), \Delta\left(w-w_{n}\right)\right\rangle .
$$

Consequently,

$$
\left\langle u-u_{n}, g\right\rangle \leq\left\|\Delta\left(u-u_{n}\right)\right\|_{L_{2}(\Omega)}\left\|\Delta\left(w-w_{n}\right)\right\|_{L_{2}(\Omega)} .
$$

This in connection with (3.8) and (3.10) gives

$$
\left\langle u-u_{n}, g\right\rangle \leq C^{2} h^{4}\|f\|_{L_{2}(\Omega)}\|g\|_{L_{2}(\Omega)} .
$$

The above inequality is valid for all $g \in L_{2}(\Omega)$. Therefore, by the converse to the Hölder inequality, we obtain

$$
\left\|u-u_{n}\right\|_{L_{2}(\Omega)} \leq C^{2} h^{4}\|f\|_{L_{2}(\Omega)} .
$$

It follows from (3.8) that

$$
\left|u-u_{n}\right|_{H^{2}(\Omega)}=\left\|\Delta\left(u-u_{n}\right)\right\|_{L_{2}(\Omega)} \leq C h^{2}\|f\|_{L_{2}(\Omega)} .
$$

Finally, by the interpolation theorem we have

$$
\left|u-u_{n}\right|_{H^{1}(\Omega)} \leq C_{1}\left\|u-u_{n}\right\|_{L_{2}(\Omega)}^{1 / 2}\left|u-u_{n}\right|_{H^{2}(\Omega)}^{1 / 2} \leq C_{2} h^{3}\|f\|_{L_{2}(\Omega)},
$$

where $C_{1}$ and $C_{2}$ are constants independent of $f$ and $n$. 


\section{Norm EQUivalence}

For the shift-invariant case, norm equivalence based on wavelet decompositions was investigated in [26, Chap. 6] and [11. The following discussions on norm equivalence are pertinent to our purpose.

For $y=\left(y_{1}, \ldots, y_{s}\right) \in \mathbb{R}^{s}$, let $|y|:=\left|y_{1}\right|+\cdots+\left|y_{s}\right|$. For $x, y \in \mathbb{R}^{s}$, we use $[x, y]$ to denote the line segment $\{(1-t) x+t y: 0 \leq t \leq 1\}$. Given a nonempty open subset $\Omega$ of $\mathbb{R}^{s}$ and $y \in \mathbb{R}^{s}$, we set $\Omega_{y}:=\{x \in \Omega:[x-y, x] \subset \Omega\}$. The modulus of continuity of a function $f$ in $L_{p}(\Omega)$ for $1 \leq p<\infty$, or $f \in C(\Omega)$ for $p=\infty$, is defined by

$$
\omega(f, h)_{p}:=\sup _{|y| \leq h}\left\|\nabla_{y} f\right\|_{p, \Omega_{y}}, \quad h>0 .
$$

For a positive integer $m$, the $m$ th modulus of smoothness of $f$ is defined by

$$
\omega_{m}(f, h)_{p}:=\sup _{|y| \leq h}\left\|\nabla_{y}^{m} f\right\|_{p, \Omega_{m y}}, \quad h>0 .
$$

For $\mu>0$ and $1 \leq p, q \leq \infty$, the Besov space $B_{p, q}^{\mu}(\Omega)$ is the collection of those functions $f \in L_{p}(\Omega)$ for which the following semi-norm is finite:

$$
|f|_{B_{p, q}^{\mu}(\Omega)}:= \begin{cases}\left(\sum_{k \in \mathbb{Z}}\left[2^{k \mu} \omega_{m}\left(f, 2^{-k}\right)_{p}\right]^{q}\right)^{1 / q}, & \text { for } 1 \leq q<\infty, \\ \sup _{k \in \mathbb{Z}}\left\{2^{k \mu} \omega_{m}\left(f, 2^{-k}\right)_{p}\right\}, & \text { for } q=\infty,\end{cases}
$$

where $m$ is the least integer greater than $\mu$. The norm for $B_{p, q}^{\mu}(\Omega)$ is

$$
\|f\|_{B_{p, q}^{\mu}(\Omega)}:=\|f\|_{L_{p}(\Omega)}+|f|_{B_{p, q}^{\mu}(\Omega)} .
$$

If $p=q=2$, then the Besov space $B_{2,2}^{\mu}\left(\mathbb{R}^{s}\right)$ is the same as the Sobolev space $H^{\mu}\left(\mathbb{R}^{s}\right)$, and the semi-norms $|\cdot|_{B_{2,2}^{\mu}}$ and $|\cdot|_{H^{\mu}}$ are equivalent (see [29, Chap. 5]).

A function $f$ in $C_{c}^{\infty}(\Omega)$ can be extended to a function in $C_{c}^{\infty}\left(\mathbb{R}^{s}\right)$ by setting $f(x)=0$ for $x \in \mathbb{R}^{s} \backslash \Omega$. In this way, $C_{c}^{\infty}(\Omega)$ can be regarded as a subspace of $C_{c}^{\infty}\left(\mathbb{R}^{s}\right)$. We use $H_{0}^{\mu}(\Omega)$ to denote the closure of $C_{c}^{\infty}(\Omega)$ in $H^{\mu}\left(\mathbb{R}^{s}\right)$.

For $n \geq 3$, let $V_{n}(0,1)$ be the linear space of splines given in $\S 2$. Then $V_{n}(0,1)$ is a closed subspace of $H_{0}^{\mu}(0,1)$ for $0 \leq \mu<5 / 2$. Let $Q_{n}$ be the operator given in (2.1). For $n \geq 3,\left.Q_{n}\right|_{L_{2}(0,1)}$ is a projection from $L_{2}(0,1)$ onto $V_{n}(0,1)$. We have the following estimate (see [16] and [19]):

$$
\left\|f-Q_{n} f\right\|_{2} \leq C \omega_{4}\left(f, 1 / 2^{n}\right)_{2} \quad \forall f \in L_{2}(0,1),
$$

where $C$ is a constant independent of $n$ and $f$.

Theorem 4.1. For $n=3,4, \ldots$, let $Q_{n}$ be the operator given in (2.1). Then there exist two positive constants $C_{1}$ and $C_{2}$ such that the inequalities

$$
\begin{aligned}
C_{1}|f|_{H_{0}^{\mu}(0,1)} & \leq\left(\left[2^{3 \mu}\left\|Q_{3} f\right\|_{2}\right]^{2}+\sum_{n=4}^{\infty}\left[2^{n \mu}\left\|\left(Q_{n}-Q_{n-1}\right) f\right\|_{2}\right]^{2}\right)^{1 / 2} \\
& \leq C_{2}|f|_{H_{0}^{\mu}(0,1)}
\end{aligned}
$$

hold for all $f \in H_{0}^{\mu}(0,1)$ with $0<\mu<5 / 2$.

Proof. Given $f \in L_{2}(0,1)$, we write $f_{n}:=Q_{n} f$ for $n \geq 3$. Let $g_{3}:=f_{3}$ and $g_{n}:=f_{n}-f_{n-1}$ for $n \geq 4$. Then

$$
f=\sum_{n=3}^{\infty} g_{n}
$$


where the series converges in $L_{2}(0,1)$. In what follows we use $C_{i}(i \in \mathbb{N})$ to denote a positive constant independent of $f$ and $n$.

For the first inequality in (4.2), we express each $g_{n}$ as

$$
g_{n}=\sum_{k=1}^{2^{n}-1} b_{n, k} \phi_{n, k}, \quad n=3,4, \ldots,
$$

where $b_{n, k} \in \mathbb{C}\left(k=1, \ldots, 2^{n}-1\right)$. Note that $\left(2^{-n \mu} \phi_{n, k}\right)_{n=3,4, \ldots, k=1, \ldots, 2^{n}-1}$ is a Bessel sequence in $H_{0}^{\mu}(0,1)$ (see Theorem 1.2 of [17]). Accordingly,

$$
|f|_{H_{0}^{\mu}(0,1)}=\left|\sum_{n=3}^{\infty} \sum_{k=1}^{2^{n}-1} 2^{n \mu} b_{n, k} 2^{-n \mu} \phi_{n, k}\right|_{H_{0}^{\mu}(0,1)} \leq C_{1}\left(\sum_{n=3}^{\infty} \sum_{k=1}^{2^{n}-1}\left|2^{n \mu} b_{n, k}\right|^{2}\right)^{1 / 2} .
$$

Since $\left\{\phi_{n, k}: 1 \leq k \leq 2^{n}-1\right\}$ is a Riesz basis of $V_{n}(0,1)$ with Riesz bounds independent of $n$, it follows from (4.3) that

$$
\sum_{k=1}^{2^{n}-1}\left|b_{n, k}\right|^{2} \leq C_{2}^{2}\left\|g_{n}\right\|_{2}^{2}
$$

Consequently,

$$
|f|_{H_{0}^{\mu}(0,1)} \leq C_{1} C_{2}\left(\sum_{n=3}^{\infty}\left[2^{n \mu}\left\|g_{n}\right\|_{2}\right]^{2}\right)^{1 / 2} .
$$

For the second inequality in (4.2), we deduce from (4.1) that

$$
\left\|g_{n}\right\|_{2}=\left\|Q_{n} f-Q_{n-1} f\right\|_{2} \leq\left\|Q_{n} f-f\right\|_{2}+\left\|Q_{n-1} f-f\right\|_{2} \leq C_{3} \omega_{4}\left(f, 1 / 2^{n}\right)_{2} .
$$

Consequently,

$$
\left(\sum_{n=4}^{\infty}\left[2^{n \mu}\left\|g_{n}\right\|_{2}\right]^{2}\right)^{1 / 2} \leq C_{3}\left(\sum_{n=4}^{\infty}\left[2^{n \mu} \omega_{4}\left(f, 1 / 2^{n}\right)_{2}\right]^{2}\right)^{1 / 2} \leq C_{4}|f|_{H_{0}^{\mu}(0,1)} .
$$

Moreover, for $f \in H_{0}^{\mu}(0,1)$, by Poincaré's inequality we have

$$
\left\|Q_{3} f\right\|_{2} \leq\left\|Q_{3}\right\|\|f\|_{2} \leq C_{5}|f|_{H_{0}^{\mu}(0,1)} .
$$

This completes the proof of (4.2).

For $n \geq 3$, let $V_{n}\left((0,1)^{2}\right)$ be the linear space of splines given in $\S 2$. Then $V_{n}\left((0,1)^{2}\right)$ is a closed subspace of $H_{0}^{\mu}\left((0,1)^{2}\right)$ for $0 \leq \mu<5 / 2$. Let $\mathcal{Q}_{n}$ be the operator given in (2.3). For $n \geq 3,\left.\mathcal{Q}_{n}\right|_{L_{2}\left((0,1)^{2}\right)}$ is a projection from $L_{2}\left((0,1)^{2}\right)$ onto $V_{n}\left((0,1)^{2}\right)$. We have the following estimate (see [16] and [19]):

$$
\left\|f-\mathcal{Q}_{n} f\right\|_{2} \leq C \omega_{4}\left(f, 1 / 2^{n}\right)_{2} \quad \forall f \in L_{2}\left((0,1)^{2}\right),
$$

where $C$ is a constant independent of $n$ and $f$. By using the same argument as in the proof of the above theorem, and taking (4.4) into account, we obtain the following theorem on norm equivalence for the space $H_{0}^{\mu}\left((0,1)^{2}\right)$.

Theorem 4.2. For $n=3,4, \ldots$, let $\mathcal{Q}_{n}$ be the operator given in (2.3). Then there exist two positive constants $C_{1}$ and $C_{2}$ such that the inequalities

$C_{1}|f|_{H_{0}^{\mu}\left((0,1)^{2}\right)} \leq\left(\left[2^{3 \mu}\left\|\mathcal{Q}_{3} f\right\|_{2}\right]^{2}+\sum_{n=4}^{\infty}\left[2^{n \mu}\left\|\left(\mathcal{Q}_{n}-\mathcal{Q}_{n-1}\right) f\right\|_{2}\right]^{2}\right)^{1 / 2} \leq C_{2}|f|_{H_{0}^{\mu}\left((0,1)^{2}\right)}$

hold for all $f \in H_{0}^{\mu}\left((0,1)^{2}\right)$ with $0<\mu<5 / 2$. 
As a consequence of Theorems 4.1 and 4.2 , we see that $|\cdot|_{H_{0}^{\mu}(0,1)}$ and $|\cdot|_{H_{0}^{\mu}\left((0,1)^{2}\right)}$ are norms.

\section{Wavelet Bases in Hilbert SPACES}

In this section, we investigate Riesz bases of Hilbert spaces equipped with some induced norms. The reader is referred to [33, [30, and [21] for related work.

Let $J$ be a countable set. For a complex-valued sequence $u=\left(u_{j}\right)_{j \in J}$, let

$$
\|u\|_{p}:=\left(\sum_{j \in J}\left|u_{j}\right|^{p}\right)^{1 / p} \quad \text { for } 1 \leq p<\infty,
$$

and let $\|u\|_{\infty}$ denote the supremum of $\left\{\left|u_{j}\right|: j \in J\right\}$. For $1 \leq p \leq \infty$, by $\ell_{p}(J)$ we denote the Banach space of all sequences $u$ on $J$ such that $\|u\|_{p}<\infty$. In particular, $\ell_{2}(J)$ is a Hilbert space with the inner product given by $\langle u, v\rangle:=\sum_{j \in J} u_{j} \overline{v_{j}}$ for $u, v \in \ell_{2}(J)$.

Let $A=\left(a_{j k}\right)_{j, k \in J}$ be a square matrix with its entries being complex numbers. For a sequence $y=\left(y_{k}\right)_{k \in J}, A y$ is the sequence $z=\left(z_{j}\right)_{j \in J}$ given by $z_{j}:=\sum_{k \in J} a_{j k} y_{k}, j \in J$, provided the above series converges absolutely for every $j \in J$. If $A$ is a bounded operator on $\ell_{p}(J)$ for some $p, 1 \leq p \leq \infty$, then the norm of $A$ on $\ell_{p}(J)$ is defined by $\|A\|_{p}:=\sup _{\|y\|_{p} \leq 1}\|A y\|_{p}$. It is easily seen that

$$
\|A\|_{1}=\sup _{k \in J} \sum_{j \in J}\left|a_{j k}\right|, \quad\|A\|_{\infty}=\sup _{j \in J} \sum_{k \in J}\left|a_{j k}\right| \quad \text { and } \quad\|A\|_{2} \leq\|A\|_{1}^{1 / 2}\|A\|_{\infty}^{1 / 2} .
$$

Let $H$ be a Hilbert space. Suppose that $V_{0}=\{0\}$ and $\left(V_{n}\right)_{n=1,2, \ldots}$ is a nested family of closed subspaces of $H: V_{n} \subset V_{n+1}$ for all $n \in \mathbb{N}$. Assume that $\bigcup_{n=1}^{\infty} V_{n}$ is dense in $H$. Then every element $f \in H$ can be represented as a convergent series $\sum_{n=1}^{\infty} f_{n}$ in $H$ with $f_{n} \in V_{n}$ for each $n \in \mathbb{N}$.

Fix $\mu>0$ and let $H_{\mu}$ be a linear subspace of $H$. Suppose that $H_{\mu}$ itself is a normed linear space. We assume that there are two positive constants $A_{1}$ and $A_{2}$ such that the following two statements are valid:

(a) If $f \in H_{\mu}$ has a decomposition $f=\sum_{n=1}^{\infty} f_{n}$ with $f_{n} \in V_{n}$, then

$$
\|f\|_{H_{\mu}} \leq A_{1}\left(\sum_{n=1}^{\infty}\left[2^{n \mu}\left\|f_{n}\right\|\right]^{2}\right)^{1 / 2} .
$$

(b) For each $f \in H_{\mu}$, there exists a decomposition $f=\sum_{n=1}^{\infty} f_{n}$ with $f_{n} \in V_{n}$ such that

$$
\left(\sum_{n=1}^{\infty}\left[2^{n \mu}\left\|f_{n}\right\|\right]^{2}\right)^{1 / 2} \leq A_{2}\|f\|_{H_{\mu}}
$$

By Theorems 4.1 and 4.2 , for $0<\mu<5 / 2$, the space $H_{0}^{\mu}(0,1)$ relative to $L_{2}(0,1)$, and the space $H_{0}^{\mu}\left((0,1)^{2}\right)$ relative to $L_{2}\left((0,1)^{2}\right)$ satisfy the above conditions.

Suppose $f \in V_{n}$. We claim that there exists a decomposition $f=\sum_{m=1}^{n} g_{m}$ with $g_{m} \in V_{m}(m=1, \ldots, n)$ such that

$$
\left(\sum_{m=1}^{n}\left[2^{m \mu}\left\|g_{m}\right\|\right]^{2}\right)^{1 / 2} \leq A_{3}\|f\|_{H_{\mu}},
$$


where $A_{3}$ is a constant independent of $f$ and $n$. Indeed, there exists a decomposition $f=\sum_{n=1}^{\infty} f_{n}$ with $f_{n} \in V_{n}(n \in \mathbb{N})$ such that (5.3) is valid. Let $g_{m}:=f_{m} \in V_{m}$ for $m=1, \ldots, n-1$ and $g_{n}:=f-\sum_{m=1}^{n-1} f_{m}$. Clearly, $g_{n} \in V_{n}$. We have

$$
\left\|g_{n}\right\|=\left\|\sum_{m=n}^{\infty} f_{m}\right\| \leq \sum_{m=n}^{\infty}\left\|f_{m}\right\| \leq\left(\sum_{m=n}^{\infty}\left[2^{-m \mu}\right]^{2}\right)^{1 / 2}\left(\sum_{m=n}^{\infty}\left[2^{m \mu}\left\|f_{m}\right\|\right]^{2}\right)^{1 / 2},
$$

where the Schwarz inequality has been used to derive the last inequality. Consequently,

$$
\sum_{m=1}^{n}\left[2^{m \mu}\left\|g_{m}\right\|\right]^{2}=\sum_{m=1}^{n-1}\left[2^{m \mu}\left\|g_{m}\right\|\right]^{2}+\left[2^{n \mu}\left\|g_{n}\right\|\right]^{2} \leq \frac{1}{1-2^{-2 \mu}} \sum_{m=1}^{\infty}\left[2^{m \mu}\left\|f_{m}\right\|\right]^{2} .
$$

This together with (5.3) establishes the desired inequality (5.4) with $A_{3}:=$ $A_{2} / \sqrt{1-2^{-2 \mu}}$.

For $n \in \mathbb{N}_{0}$, let $P_{n}$ be a linear projection from $V_{n+1}$ onto $V_{n}$, and let $W_{n}$ be the kernel space of $P_{n}$. Then $V_{n+1}$ is the direct sum of $V_{n}$ and $W_{n}$. Let $\left\{\psi_{n, k}: k \in K_{n}\right\}$ be a Riesz basis of $W_{n}$ with Riesz bounds independent of $n$.

Theorem 5.1. For $\mu>0,\left(2^{-n \mu} \psi_{n, k}\right)_{n \in \mathbb{N}_{0}, k \in K_{n}}$ is a Bessel sequence in $H_{\mu}$.

Proof. Suppose $f=\sum_{n=0}^{\infty} g_{n}$, where

$$
g_{n}=\sum_{k \in K_{n}} b_{n, k} 2^{-n \mu} \psi_{n, k} \in V_{n+1} .
$$

Since $\left\{\psi_{n, k}: k \in K_{n}\right\}$ is a Riesz basis of $W_{n}$ with Riesz bounds independent of $n$, there exists a constant $C_{1}$ independent of $n$ such that

$$
\left\|\sum_{k \in K_{n}} b_{n, k} \psi_{n, k}\right\|^{2} \leq C_{1}^{2} \sum_{k \in K_{n}}\left|b_{n, k}\right|^{2} .
$$

This in connection with (5.2) yields

$$
\|f\|_{H_{\mu}}^{2} \leq A_{1}^{2} \sum_{n=0}^{\infty}\left[2^{(n+1) \mu}\left\|g_{n}\right\|\right]^{2}=2^{2 \mu} A_{1}^{2} \sum_{n=0}^{\infty}\left\|\sum_{k \in K_{n}} b_{n, k} \psi_{n, k}\right\|^{2} \leq C_{2}^{2} \sum_{n=0}^{\infty} \sum_{k \in K_{n}}\left|b_{n, k}\right|^{2},
$$

where $C_{2}:=2^{\mu} A_{1} C_{1}$. Hence, $\left(2^{-n \mu} \psi_{n, k}\right)_{n \in \mathbb{N}_{0}, k \in K_{n}}$ is a Bessel sequence in $H_{\mu}$.

Fix $n \in \mathbb{N}$ for the time being. For each $m$ with $0 \leq m \leq n$, let $T_{m}$ be a linear projection from $V_{n}$ onto $V_{m}$. In particular, $T_{0}=0$, and $T_{n}$ is the identity operator on $V_{n}$.

Theorem 5.2. Suppose that for $j \in[m, n] \cap \mathbb{N}_{0},\left\|T_{m} f\right\| \leq B 2^{\nu(j-m)}\|f\|$ for all $f \in V_{j}$, where $0<\nu<\mu$ and $B$ is a constant independent of $m, j$, and $f$. Then

$$
\left(\sum_{m=1}^{n}\left[2^{\mu m}\left\|\left(T_{m}-T_{m-1}\right) f\right\|\right]^{2}\right)^{1 / 2} \leq C\|f\|_{H_{\mu}} \quad \forall f \in V_{n}
$$

where $C$ is a constant depending on $\mu, \nu$, and $B$ only.

Proof. Let $f \in V_{n}$. Then there exists a decomposition $f=\sum_{m=1}^{n} g_{m}$ with $g_{m} \in V_{m}$ for each $m$ such that (5.4) is valid. For $j<m$, we have $\left(T_{m}-T_{m-1}\right) g_{j}=0$, and hence

$$
\left(T_{m}-T_{m-1}\right) f=\sum_{j=m}^{n}\left(T_{m}-T_{m-1}\right) g_{j} .
$$


For $m \leq j \leq n$, we have

$$
\left\|\left(T_{m}-T_{m-1}\right) g_{j}\right\| \leq\left\|T_{m} g_{j}\right\|+\left\|T_{m-1} g_{j}\right\| \leq B\left(1+2^{\nu}\right) 2^{\nu(j-m)}\left\|g_{j}\right\| .
$$

It follows that

$$
2^{\mu m}\left\|\left(T_{m}-T_{m-1}\right) f\right\| \leq B\left(1+2^{\nu}\right) \sum_{j=m}^{n} 2^{-(\mu-\nu)(j-m)}\left[2^{j \mu}\left\|g_{j}\right\|\right] .
$$

Write $y_{m}:=2^{\mu m}\left\|\left(T_{m}-T_{m-1}\right) f\right\|$ for $m=1, \ldots, n$ and $z_{j}:=B\left(1+2^{\nu}\right)\left[2^{j \mu}\left\|g_{j}\right\|\right]$ for $j=1, \ldots, n$. Then $y_{m} \leq \sum_{j=1}^{n} a_{m j} z_{j}$ for $m=1, \ldots, n$, where $a_{m j}:=2^{-(\mu-\nu)(j-m)}$ for $m \leq j \leq n$ and 0 otherwise. Let $A$ denote the matrix $\left(a_{m j}\right)_{1 \leq m, j \leq n}$. It is easily seen that $\|A\|_{1} \leq 1 /\left(1-2^{-(\mu-\nu)}\right)$ and $\|A\|_{\infty} \leq 1 /\left(1-2^{-(\mu-\nu)}\right)$. Hence, by (5.1) we obtain $\|A\|_{2} \leq 1 /\left(1-2^{-(\mu-\nu)}\right)$. Consequently,

$$
\left(\sum_{m=1}^{n}\left[2^{\mu m}\left\|\left(T_{m}-T_{m-1}\right) f\right\|\right]^{2}\right)^{1 / 2} \leq \frac{B\left(1+2^{\nu}\right)}{1-2^{-(\mu-\nu)}}\left(\sum_{j=1}^{n}\left[2^{j \mu}\left\|g_{j}\right\|\right]^{2}\right)^{1 / 2} .
$$

This in connection with (5.4) yields the desired inequality (5.5).

Theorem 5.3. Suppose that $0<\nu<\mu$ and there exists a positive constant $B$ such that

$$
\left\|P_{m} \cdots P_{n-1}\right\| \leq B 2^{\nu(n-m)}
$$

for all $m, n \in \mathbb{N}$ with $m<n$. Then $\left\{2^{-m \mu} \psi_{m, k}: m \in \mathbb{N}_{0}, k \in K_{m}\right\}$ is a Riesz basis of $H_{\mu}$.

Proof. By Theorem 5.1, $\left\{2^{-m \mu} \psi_{m, k}: m \in \mathbb{N}_{0}, k \in K_{m}\right\}$ is a Bessel sequence in $H_{\mu}$. In order to prove that it is a Riesz basis of $H_{\mu}$, let $f$ be an element in $H_{\mu}$ given by

$$
f=\sum_{j=0}^{n-1} \sum_{k \in K_{j}} b_{j, k} 2^{-j \mu} \psi_{j, k}
$$

For $m=0,1, \ldots, n-1$, let $T_{m}:=P_{m} \cdots P_{n-1}$, and let $T_{n}$ be the identity operator on $V_{n}$. If $m<j \leq n$ and $f \in V_{j}$, then $\left\|T_{m} f\right\|=\left\|P_{m} \cdots P_{j-1} f\right\| \leq B 2^{\nu(j-m)}\|f\|$, by the hypothesis of the theorem. Since $T_{m} \psi_{j, k}=0$ for $m \leq j \leq n-1$, it follows from (5.6) that

$$
T_{m} f=\sum_{j=0}^{m-1} \sum_{k \in K_{j}} b_{j, k} 2^{-j \mu} \psi_{j, k}
$$

Hence,

$$
T_{m+1} f-T_{m} f=\sum_{k \in K_{m}} b_{m, k} 2^{-m \mu} \psi_{m, k} .
$$

Since $\left\{\psi_{m, k}: k \in K_{m}\right\}$ is a Riesz basis of $W_{m}$ with Riesz bounds independent of $m$, there exists a positive constant $C_{1}$ independent of $m$ such that

$$
\left(\sum_{k \in K_{m}}\left|2^{-m \mu} b_{m, k}\right|^{2}\right)^{1 / 2} \leq C_{1}\left\|\left(T_{m+1}-T_{m}\right) f\right\| .
$$

Consequently, by Theorem 5.2 we obtain

$$
\left(\sum_{m=0}^{n-1} \sum_{k \in K_{m}}\left|b_{m, k}\right|^{2}\right)^{1 / 2} \leq C_{1}\left(\sum_{m=0}^{n-1}\left[2^{m \mu}\left\|\left(T_{m+1}-T_{m}\right) f\right\|\right]^{2}\right)^{1 / 2} \leq C_{2}\|f\|_{H_{\mu}},
$$


where $C_{2}$ is a constant independent of $f$. This shows that $\left\{2^{-m \mu} \psi_{m, k}: m \in \mathbb{N}_{0}, k \in\right.$ $\left.K_{m}\right\}$ is a Riesz sequence in $H_{\mu}$.

It remains to show that $\bigcup_{n=1}^{\infty} V_{n}$ is dense in $H_{\mu}$. Suppose $f \in H_{\mu}$. Then $f$ has a decomposition $f=\sum_{n=1}^{\infty} f_{n}$ with $f_{n} \in V_{n}$ such that (5.3) is valid. Let $g_{n}:=\sum_{m=1}^{n} f_{m}$ for $n \in \mathbb{N}$. Clearly, $g_{n} \in V_{n}$. It follows from (5.2) that

$$
\left\|f-g_{n}\right\|_{H_{\mu}} \leq A_{1}\left(\sum_{m=n+1}^{\infty}\left[2^{m \mu}\left\|f_{m}\right\|\right]^{2}\right)^{1 / 2} .
$$

By (5.3), the series $\sum_{n=1}^{\infty}\left[2^{n \mu}\left\|f_{n}\right\|\right]^{2}$ converges. Therefore, $\lim _{n \rightarrow \infty}\left\|f-g_{n}\right\|_{H_{\mu}}=0$, as desired.

\section{WAVELET BASES IN $H^{\mu}(0,1)$ AND $H^{\mu}\left((0,1)^{2}\right)$}

In this section we use the cubic splines studied in $\S 2$ to construct wavelet bases of $H_{0}^{\mu}(0,1)$ and $H_{0}^{\mu}\left((0,1)^{2}\right)$.

For $j \in \mathbb{Z}$, let $\phi_{j}$ be the B-splines given in $\S 2$. Recall that $\phi_{n, j}(x)=2^{n / 2} \phi_{j}\left(2^{n} x\right)$, $x \in \mathbb{R}$. For $n \geq 3,\left\{\phi_{n, j}: j=1, \ldots, 2^{n}-1\right\}$ is a Riesz basis of $V_{n}(0,1)$ with Riesz bounds independent of $n$. Suppose $f_{n}=\sum_{j=1}^{2^{n}-1} a_{n, j} \phi_{n, j} \in V_{n}(0,1)$. Then

$$
B_{1}^{2} \sum_{j=1}^{2^{n}-1}\left|a_{n, j}\right|^{2} \leq\left\|\sum_{j=1}^{2^{n}-1} a_{n, j} \phi_{n, j}\right\|_{2}^{2} \leq B_{2}^{2} \sum_{j=1}^{2^{n}-1}\left|a_{n, j}\right|^{2},
$$

where $B_{1}$ and $B_{2}$ are positive constants independent of $n$. We have

$$
\sum_{j=1}^{2^{n}-1} a_{n, j} \phi_{n, j}\left(k / 2^{n}\right)=f_{n}\left(k / 2^{n}\right) \quad \text { for } k=1, \ldots, 2^{n}-1 .
$$

Note that $\phi_{n, j}\left(k / 2^{n}\right)=2^{n / 2} \phi_{j}(k)$. Let $F_{n}$ denote the matrix $\left(\phi_{j}(k)\right)_{1 \leq j, k \leq 2^{n}-1}$. Then

$$
F_{n}=\left[\begin{array}{ccccccc}
7 / 12 & 1 / 6 & 0 & \cdots & 0 & 0 & 0 \\
1 / 6 & 2 / 3 & 1 / 6 & \cdots & 0 & 0 & 0 \\
\vdots & \vdots & \vdots & & \vdots & \vdots & \vdots \\
0 & 0 & 0 & \cdots & 1 / 6 & 2 / 3 & 1 / 6 \\
0 & 0 & 0 & \cdots & 0 & 1 / 6 & 7 / 12
\end{array}\right] .
$$

Let $\left\|F_{n}\right\|$ denote the norm of the matrix $F_{n}$ on the $\ell_{2}$ space. Clearly, $\left\|F_{n}\right\| \leq 1$. Moreover, $F_{n}$ is diagonally dominant. It is easily verified that $\left\|F_{n}^{-1}\right\| \leq 3$ for all $n \geq 3$. Consequently,

$$
C_{1}^{2} \sum_{k=1}^{2^{n}-1}\left|f_{n}\left(k / 2^{n}\right)\right|^{2} \leq \sum_{j=1}^{2^{n}-1}\left|2^{n / 2} a_{n, j}\right|^{2} \leq C_{2}^{2} \sum_{k=1}^{2^{n}-1}\left|f_{n}\left(k / 2^{n}\right)\right|^{2},
$$

where $C_{1}$ and $C_{2}$ are positive constants independent of $n$.

Let $P_{n}$ be the linear projection from $V_{n+1}$ onto $V_{n}$ given as follows: For $f_{n+1} \in$ $V_{n+1}, f_{n}:=P_{n} f_{n+1}$ is the unique element in $V_{n}(0,1)$ determined by the interpolation condition

$$
f_{n}\left(k / 2^{n}\right)=f_{n+1}\left(k / 2^{n}\right) \quad \forall k=1, \ldots, 2^{n}-1 .
$$

Suppose $f_{n}=\sum_{j=1}^{2^{n}-1} a_{n, j} \phi_{n, j} \in V_{n}$. For $m \geq 3$, let $f_{m}:=P_{m} \cdots P_{n-1} f_{n}$. The function $f_{m}$ can be represented as $f_{m}=\sum_{j=1}^{2^{m}-1} a_{m, j} \phi_{m, j}$. We have $f_{m}\left(k / 2^{m}\right)=$ 
$f_{n}\left(k / 2^{m}\right)$ for $k=1, \ldots, 2^{m}-1$. It follows from (6.1) and (6.2) that

$$
\begin{aligned}
\left\|f_{m}\right\|_{2}^{2} & \leq B_{2}^{2} \sum_{j=1}^{2^{m}-1}\left|a_{m, j}\right|^{2} \leq B_{2}^{2} C_{2}^{2} 2^{-m} \sum_{k=1}^{2^{m}-1}\left|f_{m}\left(k / 2^{m}\right)\right|^{2} \\
& \leq B_{2}^{2} C_{2}^{2} 2^{-m} \sum_{k=1}^{2^{n}-1}\left|f_{n}\left(k / 2^{n}\right)\right|^{2} \leq B_{2}^{2} C_{2}^{2} C_{1}^{-2} 2^{-m} \sum_{j=1}^{2^{n}-1}\left|2^{n / 2} a_{n, j}\right|^{2} \\
& \leq B_{2}^{2} C_{2}^{2} C_{1}^{-2} B_{1}^{-2} 2^{n-m}\left\|f_{n}\right\|_{2}^{2} .
\end{aligned}
$$

Consequently,

$$
\left\|P_{m} \cdots P_{n-1}\right\| \leq C 2^{(n-m) / 2},
$$

where $C:=B_{2} C_{2} C_{1}^{-1} B_{1}^{-1}$.

The wavelets are constructed as follows. Let

$$
\psi(x):=-\frac{1}{4} \phi(2 x)+\phi(2 x-1)-\frac{1}{4} \phi(2 x-2), \quad x \in \mathbb{R} .
$$

Then $\psi$ is supported on $[0,3]$. Moreover,

$$
\psi(1)=-\frac{1}{4} \phi(2)+\phi(1)=0 \quad \text { and } \quad \psi(2)=\phi(3)-\frac{1}{4} \phi(2)=0 .
$$

Hence, $\psi(j)=0$ for all $j \in \mathbb{Z}$. Moreover, let

$$
\psi_{b}(x):=\phi_{b}(2 x)-\frac{1}{4} \phi(2 x), \quad x \in \mathbb{R} .
$$

Then $\psi_{b}$ is supported on $[0,2]$, and $\psi_{b}(j)=0$ for all $j \in \mathbb{Z}$. For $n \geq 3$ and $x \in \mathbb{R}$, let

$$
\psi_{n, j}(x):= \begin{cases}2^{n / 2} \psi_{b}\left(2^{n} x\right), & j=1, \\ 2^{n / 2} \psi\left(2^{n} x-j+2\right), & j=2, \ldots, 2^{n}-1, \\ 2^{n / 2} \psi_{b}\left(2^{n}(1-x)\right), & j=2^{n} .\end{cases}
$$

We have $\psi_{n, j} \in V_{n+1}(0,1)$ and $P_{n} \psi_{n, j}=0$ for $j=1, \ldots, 2^{n}$. Let $W_{n}$ be the linear span of $\left\{\psi_{n, j}: j=1, \ldots, 2^{n}\right\}$. Then $\operatorname{dim}\left(W_{n}\right)=2^{n}$. Moreover, $\left\{\psi_{n, j}: j=\right.$ $\left.1, \ldots, 2^{n}\right\}$ is a Riesz basis of $W_{n}$ with Riesz bounds independent of $n$. Thus, $W_{n}$ is the kernel space of $P_{n}$, and hence $V_{n+1}$ is the direct sum of $V_{n}$ and $W_{n}$. An application of Theorem 5.3 with $\nu=1 / 2$ gives the following result.

Theorem 6.1. For $1 / 2<\mu<5 / 2$, the set

$$
\left\{2^{-3 \mu} \phi_{3, j}: j=1, \ldots, 7\right\} \cup\left\{2^{-n \mu} \psi_{n, k}: n \geq 3, k=1, \ldots, 2^{n}\right\}
$$

forms a Riesz basis of $H_{0}^{\mu}(0,1)$.

We are in a position to investigate wavelet bases in $H_{0}^{\mu}\left((0,1)^{2}\right)$. Recall that, for $j=\left(j_{1}, j_{2}\right) \in \mathbb{Z}^{2}, \phi_{n, j}(x)=\phi_{n, j_{1}}\left(x_{1}\right) \phi_{n, j_{2}}\left(x_{2}\right), x=\left(x_{1}, x_{2}\right) \in \mathbb{R}^{2}$. For $n \geq 3$, $V_{n}\left((0,1)^{2}\right)$ is the linear span of $\left\{\phi_{n, j}: j \in J_{n}\right\}$, where $J_{n}=\left\{\left(j_{1}, j_{2}\right) \in \mathbb{Z}^{2}: 1 \leq\right.$ $\left.j_{1}, j_{2} \leq 2^{n}-1\right\}$. Clearly, $V_{n}\left((0,1)^{2}\right)$ is a subspace of $H_{0}^{\mu}\left((0,1)^{2}\right)$ for $0 \leq \mu<5 / 2$. Moreover, $\bigcup_{n=3}^{\infty} V_{n}\left((0,1)^{2}\right)$ is dense in $L_{2}\left((0,1)^{2}\right)$.

Suppose $f_{n}=\sum_{j \in J_{n}} a_{n, j} \phi_{n, j} \in V_{n}\left((0,1)^{2}\right)$. Then

$$
B_{1}^{2} \sum_{j \in J_{n}}\left|a_{n, j}\right|^{2} \leq\left\|\sum_{j \in J_{n}} a_{n, j} \phi_{n, j}\right\|_{2}^{2} \leq B_{2}^{2} \sum_{j \in J_{n}}\left|a_{n, j}\right|^{2},
$$


where $B_{1}$ and $B_{2}$ are positive constants independent of $n$. Moreover,

$$
\sum_{j \in J_{n}} a_{n, j} \phi_{n, j}\left(k / 2^{n}\right)=f_{n}\left(k / 2^{n}\right) \quad \text { for } k \in J_{n} .
$$

Note that $\phi_{n, j}\left(k / 2^{n}\right)=2^{n} \phi_{j_{1}}\left(k_{1}\right) \phi_{j_{2}}\left(k_{2}\right)$ for $j=\left(j_{1}, j_{2}\right) \in J_{n}$ and $k=\left(k_{1}, k_{2}\right) \in$ $J_{n}$. The matrix $\left(\phi_{j_{1}}\left(k_{1}\right) \phi_{j_{2}}\left(k_{2}\right)\right)_{1 \leq j_{1}, j_{2} \leq 2^{n}-1,1 \leq k_{1}, k_{2} \leq 2^{n}-1}$ can be viewed as the Kronecker product $F_{n} \otimes F_{n}$, where $F_{n}$ is the matrix $\left(\phi_{j_{1}}\left(k_{1}\right)\right)_{1 \leq j_{1}, k_{1} \leq 2^{n}-1}$. (See [15. Chap. 4] for the definition and properties of the Kronecker product.) We have

$$
\left\|F_{n} \otimes F_{n}\right\| \leq 1 \quad \text { and } \quad\left\|\left(F_{n} \otimes F_{n}\right)^{-1}\right\|=\left\|F_{n}^{-1} \otimes F_{n}^{-1}\right\| \leq 9 .
$$

Consequently,

$$
C_{1}^{2} \sum_{k \in J_{n}}\left|f_{n}\left(k / 2^{n}\right)\right|^{2} \leq \sum_{j \in J_{n}}\left|2^{n} a_{n, j}\right|^{2} \leq C_{2}^{2} \sum_{k \in J_{n}}\left|f_{n}\left(k / 2^{n}\right)\right|^{2},
$$

where $C_{1}$ and $C_{2}$ are positive constants independent of $n$. Let $P_{n}$ be the linear projection from $V_{n+1}\left((0,1)^{2}\right)$ onto $V_{n}\left((0,1)^{2}\right)$ given as follows: For $f_{n+1} \in$ $V_{n+1}\left((0,1)^{2}\right), f_{n}:=P_{n} f_{n+1}$ is the unique element in $V_{n}\left((0,1)^{2}\right)$ determined by the interpolation condition

$$
f_{n}\left(k / 2^{n}\right)=f_{n+1}\left(k / 2^{n}\right) \quad \forall k \in J_{n} .
$$

By using the same argument as above we obtain

$$
\left\|P_{m} \cdots P_{n-1}\right\| \leq C 2^{n-m},
$$

where $C$ is a constant independent of $m$ and $n$. Let $W_{n}$ be the kernel space of $P_{n}$. Then

$\operatorname{dim}\left(W_{n}\right)=\operatorname{dim}\left(V_{n+1}\left((0,1)^{2}\right)\right)-\operatorname{dim}\left(V_{n}\left((0,1)^{2}\right)\right)=\left(2^{n}-1\right) 2^{n}+2^{n}\left(2^{n}-1\right)+2^{2 n}$.

For two functions $v$ and $w$ defined on $[0,1]$, we use $v \otimes w$ to denote the function on $[0,1]^{2}$ given by $v \otimes w\left(x_{1}, x_{2}\right):=v\left(x_{1}\right) w\left(x_{2}\right), 0 \leq x_{1}, x_{2} \leq 1$. Let

$$
\begin{aligned}
\Gamma_{n}^{\prime} & :=\left\{\phi_{n, j_{1}} \otimes \psi_{n, k_{2}}: 1 \leq j_{1} \leq 2^{n}-1,1 \leq k_{2} \leq 2^{n}\right\}, \\
\Gamma_{n}^{\prime \prime} & :=\left\{\psi_{n, k_{1}} \otimes \phi_{n, j_{2}}: 1 \leq j_{2} \leq 2^{n}-1,1 \leq k_{1} \leq 2^{n}\right\}, \\
\Gamma_{n}^{\prime \prime \prime} & :=\left\{\psi_{n, k_{1}} \otimes \psi_{n, k_{2}}: 1 \leq k_{1}, k_{2} \leq 2^{n}\right\} .
\end{aligned}
$$

It is easily seen that $\Gamma_{n}:=\Gamma_{n}^{\prime} \cup \Gamma_{n}^{\prime \prime} \cup \Gamma_{n}^{\prime \prime \prime}$ is a Riesz basis of $W_{n}$ with Riesz bounds independent of $n$.

Now an application of Theorem 5.3 with $\nu=1$ gives the following result.

Theorem 6.2. For $1<\mu<5 / 2$, the set

$$
\left\{2^{-3 \mu} \phi_{3, j}: j \in J_{3}\right\} \cup \bigcup_{n=3}^{\infty}\left\{2^{-n \mu} w: w \in \Gamma_{n}\right\}
$$

forms a Riesz basis of $H_{0}^{\mu}\left((0,1)^{2}\right)$. 


\section{The WAVELET METHOD FOR THE BIHARMONIC EQUATION}

In this section we apply the wavelet bases constructed in the previous section to numerical solutions of the biharmonic equation (1.4) for $\Omega=(0,1)^{2}$. In what follows, we write $V_{n}$ for $V_{n}\left((0,1)^{2}\right)$ and always assume $n \geq 3$. Also, all the functions are real-valued.

In order to solve the variational problem $(1.5)$ on the unit square $\Omega=(0,1)^{2}$, we use $V_{n}$ to approximate $H_{0}^{2}(\Omega)$. Recall that $\Phi_{n}:=\left\{\phi_{n, j}: j \in J_{n}\right\}$ is a basis of $V_{n}$. We look for $u_{n} \in V_{n}$ such that

$$
\left\langle\Delta u_{n}, \Delta v\right\rangle=\langle f, v\rangle \quad \forall v \in V_{n}
$$

Suppose $u_{n}=\sum_{\phi \in \Phi_{n}} y_{\phi} \phi$. Let $A_{n}$ be the matrix $(\langle\Delta \sigma, \Delta \phi\rangle)_{\sigma, \phi \in \Phi_{n}}$, and let $\xi_{n}$ be the column vector $(\langle f, \phi\rangle)_{\phi \in \Phi_{n}}$. Then the column vector $y_{n}=\left(y_{\phi}\right)_{\phi \in \Phi_{n}}$ is the solution of the system of linear equations

$$
A_{n} y_{n}=\xi_{n} .
$$

However, the condition number $\kappa\left(A_{n}\right)$ of the matrix $A_{n}$ is of the size $O\left(2^{4 n}\right)$. Hence, without preconditioning it would be very difficult to solve the system of equations (7.2).

Now we employ the wavelet bases constructed in the previous section to solve the variational problem (17.1). Let $B_{n}$ be the matrix $(\langle\Delta \chi, \Delta \psi\rangle)_{\chi, \psi \in \Psi_{n}}$, where

$$
\Psi_{n}:=\left\{2^{-6} \phi_{3, j}: j \in J_{3}\right\} \cup \bigcup_{k=3}^{n-1}\left\{2^{-2 k} w: w \in \Gamma_{k}\right\} .
$$

We use $\lambda_{\max }\left(B_{n}\right)$ and $\lambda_{\min }\left(B_{n}\right)$ to denote the maximal and minimal eigenvalue of $B_{n}$, respectively. Then $\kappa\left(B_{n}\right):=\lambda_{\max }\left(B_{n}\right) / \lambda_{\min }\left(B_{n}\right)$ gives the condition number of $B_{n}$ in the 2-norm. We claim that the condition number $\kappa\left(B_{n}\right)$ is uniformly bounded (independent of $n$ ). To justify our claim, let $\lambda$ be an eigenvalue of the matrix $B_{n}$. Then there exists a nonzero column vector $z \in \mathbb{R}^{\Psi_{n}}$ such that $B_{n} z=\lambda z$. It follows that $z^{T} B_{n} z=\lambda z^{T} z$, where $z^{T}$ denotes the transpose of the column vector $z$. Suppose $z=\left(a_{\psi}\right)_{\psi \in \Psi_{n}}$. It is easily seen that

$$
z^{T} B_{n} z=\left\langle\sum_{\psi \in \Psi_{n}} a_{\psi} \Delta \psi, \sum_{\psi \in \Psi_{n}} a_{\psi} \Delta \psi\right\rangle=\left\|\Delta\left(\sum_{\psi \in \Psi_{n}} a_{\psi} \psi\right)\right\|_{L_{2}(\Omega)}^{2}=\left|\sum_{\psi \in \Psi_{n}} a_{\psi} \psi\right|_{H_{0}^{2}(\Omega)}^{2} .
$$

By Theorem 6.2, there exist two positive constants $C_{1}$ and $C_{2}$ (independent of $n$ ) such that

$$
C_{1}^{2} \sum_{\psi \in \Psi_{n}}\left|a_{\psi}\right|^{2} \leq\left|\sum_{\psi \in \Psi_{n}} a_{\psi} \psi\right|_{H_{0}^{2}(\Omega)}^{2} \leq C_{2}^{2} \sum_{\psi \in \Psi_{n}}\left|a_{\psi}\right|^{2}
$$

On the other hand, $z^{T} B_{n} z=\lambda z^{T} z=\lambda \sum_{\psi \in \Psi_{n}}\left|a_{\psi}\right|^{2}$. Consequently,

$$
C_{1}^{2} \sum_{\psi \in \Psi_{n}}\left|a_{\psi}\right|^{2} \leq \lambda \sum_{\psi \in \Psi_{n}}\left|a_{\psi}\right|^{2} \leq C_{2}^{2} \sum_{\psi \in \Psi_{n}}\left|a_{\psi}\right|^{2} .
$$

Hence, $C_{1}^{2} \leq \lambda \leq C_{2}^{2}$. This shows that $\kappa\left(B_{n}\right) \leq C_{2}^{2} / C_{1}^{2}$ for all $n \geq 3$.

The condition numbers $\kappa\left(B_{n}\right)$ are computed for $4 \leq n \leq 9$ and listed in Table 7.1. The numerical computation confirms our assertion that $\kappa\left(B_{n}\right)$ is uniformly bounded (independent of $n$ ). 
TABLE 7.1. Condition number of the preconditioned matrix

\begin{tabular}{|c|c|c|c|c|}
\hline Level $n$ & Size of $B_{n}$ & $\lambda_{\max }\left(B_{n}\right)$ & $\lambda_{\min }\left(B_{n}\right)$ & $\kappa\left(B_{n}\right)$ \\
\hline 4 & $225 \times 225$ & 2.7160 & 0.07996 & 33.97 \\
\hline 5 & $961 \times 961$ & 2.7883 & 0.07995 & 34.88 \\
\hline 6 & $3969 \times 3969$ & 2.8082 & 0.07994 & 35.13 \\
\hline 7 & $16129 \times 16129$ & 2.8259 & 0.07994 & 35.35 \\
\hline 8 & $65025 \times 65025$ & 2.8434 & 0.07994 & 35.57 \\
\hline 9 & $261121 \times 261121$ & 2.8489 & 0.07994 & 35.64 \\
\hline
\end{tabular}

The biharmonic equation (1.4) is often decoupled as

$$
-\Delta u=w \quad \text { and } \quad-\Delta w=f \quad \text { in } \Omega .
$$

For the decoupled biharmonic equation (7.5) with the homogeneous boundary condition, a multigrid preconditioner was proposed in [28. It is clear from Tables 1, 2 , and 3 in 28 that the condition number of the preconditioned matrix grows like $O\left(h^{-1}\right)$, where $h$ is the mesh size. In fact, for piecewise linear approximation on the $48 \times 48$ grid, the condition number already exceeds 80 . In comparison, the condition number of $\kappa\left(B_{n}\right)$ is uniformly bounded. For the $512 \times 512$ grid $(n=9)$, $\kappa\left(B_{9}\right)<36$.

Suppose $u_{n}=\sum_{\psi \in \Psi_{n}} z_{\psi} \psi$. Let $\eta_{n}$ be the column vector $\eta_{n}=(\langle f, \psi\rangle)_{\psi \in \Psi_{n}}$, and let $z_{n}$ be the column vector $\left(z_{\psi}\right)_{\psi \in \Psi_{n}}$. Then $u_{n}$ is the solution to the variational problem (7.1) if and only if $z_{n}$ is the solution of the following system of linear equations:

$$
B_{n} z_{n}=\eta_{n}
$$

Suppose $u_{n}^{*}=\sum_{\psi \in \Psi_{n}} z_{\psi}^{*} \psi$, where $z_{n}^{*}=\left(z_{\psi}^{*}\right)_{\psi \in \Psi_{n}}$ is the vector in $\mathbb{R}^{\Psi_{n}}$ such that $B_{n} z_{n}^{*}=\eta_{n}$, i.e., $z_{n}^{*}$ is the exact solution to the equation (7.6). Let $u$ be the exact solution to the biharmonic equation (1.4), and let $e_{n}^{*}:=u_{n}^{*}-u$. Then $\left\|\Delta e_{n}^{*}\right\|_{L_{2}(\Omega)}$ represents the discretization error in the energy norm, and $\left\|e_{n}^{*}\right\|_{L_{2}(\Omega)}$ represents the discretization error in the $L_{2}$ norm.

Suppose $u_{n}=\sum_{\psi \in \Psi_{n}} z_{\psi} \psi$, where $z_{n}=\left(z_{\psi}\right)_{\psi \in \Psi_{n}}$ is an approximate solution to the equation (7.6). Let $e_{n}:=u_{n}-u$. It was proved in Theorem 3.1 that

$$
\left\|\Delta e_{n}^{*}\right\|_{L_{2}(\Omega)}=\left\|\Delta\left(u-u_{n}^{*}\right)\right\|_{L_{2}(\Omega)} \leq\left\|\Delta\left(u-u_{n}\right)\right\|_{L_{2}(\Omega)}=\left\|\Delta e_{n}\right\|_{L_{2}(\Omega)} .
$$

Thus, for any approximate solution $u_{n}$, the error $\left\|\Delta e_{n}\right\|_{L_{2}(\Omega)}$ is no less than the discretization error $\left\|\Delta e_{n}^{*}\right\|_{L_{2}(\Omega)}$ in the energy norm. If $\left\|\Delta e_{n}\right\|_{L_{2}(\Omega)} \leq K\left\|\Delta e_{n}^{*}\right\|_{L_{2}(\Omega)}$, where $K$ is a constant close to 1 , then we say that the error of an approximate solution $u_{n}$ achieves the level of discretization error in the energy norm. Similarly, if $\left\|e_{n}\right\|_{L_{2}(\Omega)} \leq K\left\|e_{n}^{*}\right\|_{L_{2}(\Omega)}$, then we say that the error of an approximate solution $u_{n}$ achieves the level of discretization error in the $L_{2}$ norm.

We observe that each $\psi \in \Psi_{n}$ can be uniquely expressed as $\psi=\sum_{\phi \in \Phi_{n}} s_{\psi_{\phi}} \phi$. Let $S_{n}$ denote the matrix $\left(s_{\psi \phi}\right)_{\psi \in \Psi_{n}, \phi \in \Phi_{n}}$, which represents the wavelet transform. 
Then $B_{n}=S_{n} A_{n} S_{n}^{T}$ and $\eta_{n}=S_{n} \xi_{n}$. Hence, the equation (7.6) is equivalent to (7.2) if we set $y_{n}=S_{n}^{T} z_{n}$. Actually, we will use the PCG (Preconditioned Conjugate Gradient) algorithm (see, e.g., [24, pp. 94-95]) to solve the system of linear equations

$$
S_{n} A_{n} y_{n}=S_{n} \xi_{n} .
$$

We observe that $A_{n}$ is an $N^{2} \times N^{2}$ matrix and $y_{n}$ is an $N^{2}$ column vector, where $N=2^{n}-1$. Each iteration of the PCG algorithm requires a multiplication of the matrix $A_{n}$ with a vector and a multiplication of the matrix $S_{n}$ with a vector. A multiplication of $A_{n}$ with a vector requires $O\left(N^{2}\right)$ work. A multiplication of the matrix $S_{n}$ with a vector can be performed by using the corresponding wavelet transform. Hence, it also requires $O\left(N^{2}\right)$ work. We will use an approximate solution to the equation $S_{n} A_{n} y_{n}=S_{n} \xi_{n}$ as an initial guess for the equation $S_{n+1} A_{n+1} y_{n+1}=S_{n+1} \xi_{n+1}$ at the next level. Since $\kappa\left(B_{n}\right)$ is uniformly bounded, the number of iterations needed from level $n$ to level $n+1$ is bounded (independent of $n$ ). Thus, the total work required for the solution of the equation (7.8) to the level of discretization error is $O\left(N^{2}\right)$. In other words, our algorithm is optimal.

\section{NumERICAL EXAMPLES: ERROR ESTIMATES IN THE ENERGY NORM}

The biharmonic equation arises in many applications. In fluid mechanics, the solution $u$ of (1.4) represents the stream function, and $-\Delta u$ represents the vorticity of the fluid. In linear elasticity, $u$ is used to describe the airy stress function. Then $\left(D_{j} D_{k} u\right)_{1 \leq j, k \leq 2}$ gives the Cauchy stress tensor. Thus, estimation of the error $\left\|\Delta e_{n}\right\|_{L_{2}(\Omega)}$ in the energy norm has its own significance in physics. In this section, we focus on error estimates in the energy norm. Our goal is to find efficient algorithms for computing approximate solutions that achieve the level of discretization error.

For a finite nonempty subset $J, \mathbb{R}^{J}$ can be viewed as a vector space. Recall that the $\ell_{2}$ norm of a vector $z=\left(z_{j}\right)_{j \in J} \in \mathbb{R}^{J}$ is given by

$$
\|z\|_{2}=\left(\sum_{j \in J}\left|z_{j}\right|^{2}\right)^{1 / 2} .
$$

For $n \geq 3$, let $\Psi_{n}$ be the set defined in (7.3). We use $\mathcal{P}_{n}$ to denote the mapping from $\mathbb{R}^{\Psi_{n}}$ to $V_{n}$ that sends $\left(a_{\psi}\right)_{\psi \in \Psi_{n}}$ to $\sum_{\psi \in \Psi_{n}} a_{\psi} \psi$. For $z_{n} \in \mathbb{R}^{\Psi_{n}}$, it follows from (7.4) that

$$
C_{1}\left\|z_{n}\right\|_{2} \leq\left\|\Delta\left(\mathcal{P}_{n} z_{n}\right)\right\|_{L_{2}(\Omega)} \leq C_{2}\left\|z_{n}\right\|_{2} .
$$

Recall that $B_{n}$ is the matrix $(\langle\Delta \chi, \Delta \psi\rangle)_{\chi, \psi \in \Psi_{n}}$. We wish to solve the linear system of equations $B_{n} z_{n}=\eta_{n}$ with $\eta_{n}:=(\langle f, \psi\rangle)_{\psi \in \Psi_{n}}$, where $f$ is the function on the right-hand side of the biharmonic equation (1.4). For $z_{n} \in \mathbb{R}^{\Psi_{n}}, r_{n}:=\eta_{n}-B_{n} z_{n}$ represents the corresponding residue. In particular, for $z_{n}=0, r_{n}^{0}:=\eta_{n}$ is the initial residue. We have $B_{n}\left(z_{n}-z_{n}^{*}\right)=B_{n} z_{n}-\eta_{n}=-r_{n}$. Suppose that $C_{1}$ and $C_{2}$ are two positive constants such that (7.4) is valid. Then $\lambda_{\max }\left(B_{n}\right) \leq C_{2}^{2}$ and $\lambda_{\min }\left(B_{n}\right) \geq C_{1}^{2}$. Hence,

$$
C_{1}^{2}\left\|z_{n}-z_{n}^{*}\right\|_{2} \leq\left\|r_{n}\right\|_{2} \leq C_{2}^{2}\left\|z_{n}-z_{n}^{*}\right\|_{2} .
$$


This in connection with (8.1) gives

$$
\frac{C_{1}}{C_{2}^{2}}\left\|r_{n}\right\|_{2} \leq\left\|\Delta\left(u_{n}-u_{n}^{*}\right)\right\|_{L_{2}(\Omega)} \leq \frac{C_{2}}{C_{1}^{2}}\left\|r_{n}\right\|_{2},
$$

where $u_{n}=\mathcal{P}_{n} z_{n}$ and $u_{n}^{*}=\mathcal{P}_{n} z_{n}^{*}$. Recall that $e_{n}=u_{n}-u$ and $e_{n}^{*}=u^{*}-u$, where $u$ is the exact solution to the biharmonic equation (1.4). Consequently, $e_{n}-e_{n}^{*}=u_{n}-u_{n}^{*}$ and

$$
\left\|\Delta\left(e_{n}-e_{n}^{*}\right)\right\|_{L_{2}(\Omega)}=\left\|\Delta\left(u_{n}-u_{n}^{*}\right)\right\|_{L_{2}(\Omega)} \leq C\left\|r_{n}\right\|_{2},
$$

where $C=C_{2} / C_{1}^{2}$. This together with (7.7) yields

$$
\left\|\Delta e_{n}^{*}\right\|_{L_{2}(\Omega)} \leq\left\|\Delta e_{n}\right\|_{L_{2}(\Omega)} \leq\left\|\Delta e_{n}^{*}\right\|_{L_{2}(\Omega)}+C\left\|r_{n}\right\|_{2} .
$$

By Theorem 3.1, $\left\|\Delta e_{n}^{*}\right\|_{L_{2}(\Omega)} \leq M_{1} 2^{-2 n}$ for some positive constant $M_{1}$ independent of $n$. Therefore, if the residue $r_{n}$ is made so small that $\left\|r_{n}\right\|_{2} \leq M_{2} 2^{-2 n}$, we will have $\left\|\Delta e_{n}\right\|_{L_{2}(\Omega)} \leq M 2^{-2 n}$ with $M:=M_{1}+C M_{2}$.

For $k>3$, let $\mathcal{E}_{k}$ be the linear mapping from $\mathbb{R}^{\Psi_{k-1}}$ to $\mathbb{R}^{\Psi_{k}}$ that sends $\left(a_{\psi}\right)_{\psi \in \Psi_{k-1}}$ to $\left(b_{\psi}\right)_{\psi \in \Psi_{k}}$, where

$$
b_{\psi}:= \begin{cases}a_{\psi} & \text { for } \psi \in \Psi_{k-1}, \\ 0 & \text { for } \psi \in \Psi_{k} \backslash \Psi_{k-1} .\end{cases}
$$

Note that $B_{3}$ is a matrix of size $49 \times 49$. We first solve the equation $B_{3} z_{3}=\eta_{3}$ exactly and get the solution $z_{3}$. Then we use $\mathcal{E}_{4} z_{3}$ as the initial vector and perform the PCG iterations for the equation $B_{4} z_{4}=\eta_{4}$ to get an approximate solution $z_{4}$. Let $u_{3}:=\mathcal{P}_{3} z_{3}$ and $u_{4}:=\mathcal{P}_{4} u_{4}$. Then $\tilde{e}_{3}:=u_{3}-u_{4}$ represents the error in the energy norm at level 3 . For $4 \leq k \leq n$, the above discussion motivates us to choose the threshold $\varepsilon_{n, k}$ as follows:

$$
\varepsilon_{n, k}:=\frac{k}{n} \frac{\left\|\Delta \tilde{e}_{3}\right\|_{L_{2}(\Omega)}}{2^{2 n-5}} .
$$

To solve the linear system of equations $B_{n} z_{n}=\eta_{n}$ for $n \geq 4$, we will use the following multilevel algorithm based on wavelets. First, let $z_{n, 3}:=z_{3}$. Second, for $4 \leq k \leq n$, use $z_{n, k}^{0}:=\mathcal{E}_{k} z_{n, k-1}$ as the initial vector to perform the PCG iterations for the equation $B_{k} z_{n, k}=r_{k}^{0}$ sufficiently many times such that the residue $r_{n, k}:=r_{k}^{0}-B_{k} z_{n, k}$ satisfies $\left\|r_{n, k}\right\|_{2} \leq \varepsilon_{n, k}$. Finally, set $z_{n}:=z_{n, n}$ and $r_{n}:=r_{n, n}$. Then $r_{n}$ represents the residue when the algorithm stops, and $z_{n}$ is the desired approximate solution.

In the above algorithm, suppose that $m_{k}(4 \leq k \leq n)$ iterations are performed for the equation $B_{k} z_{n, k}=r_{k}^{0}$. Note that $m_{k}$ iterations at level $k$ are equivalent to $m_{k} / 4^{n-k}$ iterations at level $n$. Thus, the total number of equivalent iterations at level $n$ will be

$$
N_{i t}=\sum_{k=4}^{n} \frac{m_{k}}{4^{n-k}}
$$

We are in a position to give numerical examples to show that the above algorithm is efficient. The following computation is conducted on a Lenovo desktop with 2 GB memory and an Intel Core 2 CPU 6400 at $2.13 \mathrm{GHz}$. We implemented our algorithm in $\mathrm{C}$ and used gcc to compile it.

Example 8.1. Consider the biharmonic equation (1.4) on $\Omega$ with $f$ given by

$$
f\left(x_{1}, x_{2}\right)=t \pi^{4}\left[4 \cos \left(2 \pi x_{1}\right) \cos \left(2 \pi x_{2}\right)-\cos \left(2 \pi x_{1}\right)-\cos \left(2 \pi x_{2}\right)\right], \quad\left(x_{1}, x_{2}\right) \in \Omega,
$$


where $t>0$ is chosen so that $\|f\|_{2}=1$. The exact solution of the equation is

$$
u\left(x_{1}, x_{2}\right)=t\left[1-\cos \left(2 \pi x_{1}\right)\right]\left[1-\cos \left(2 \pi x_{2}\right)\right] / 16, \quad\left(x_{1}, x_{2}\right) \in \Omega=(0,1)^{2} .
$$

TABLE 8.1. Numerical results of Example 8.1

\begin{tabular}{|c|c|c|c|c|c|c|c|}
\hline Level $n$ & Grid $2^{n} \times 2^{n}$ & $N_{i t}$ & $\left\|r_{n}^{0}\right\|_{2}$ & $\left\|r_{n}\right\|_{2}$ & $\left\|\Delta e_{n}\right\|_{2}$ & $\left\|\Delta e_{n}^{*}\right\|_{2}$ & Time (s) \\
\hline 5 & $32 \times 32$ & 1.75 & $5.64 \mathrm{e}-3$ & $1.01 \mathrm{e}-5$ & $2.23 \mathrm{e}-5$ & $2.00 \mathrm{e}-5$ & 0.001 \\
\hline 6 & $64 \times 64$ & 1.81 & $5.64 \mathrm{e}-3$ & $1.72 \mathrm{e}-6$ & $5.21 \mathrm{e}-6$ & $4.99 \mathrm{e}-6$ & 0.002 \\
\hline 7 & $128 \times 128$ & 1.88 & $5.64 \mathrm{e}-3$ & $3.02 \mathrm{e}-7$ & $1.28 \mathrm{e}-6$ & $1.25 \mathrm{e}-6$ & 0.007 \\
\hline 8 & $256 \times 256$ & 1.80 & $5.64 \mathrm{e}-3$ & $7.25 \mathrm{e}-8$ & $3.22 \mathrm{e}-7$ & $3.11 \mathrm{e}-7$ & 0.029 \\
\hline 9 & $512 \times 512$ & 1.81 & $5.64 \mathrm{e}-3$ & $1.32 \mathrm{e}-8$ & $8.02 \mathrm{e}-8$ & $7.79 \mathrm{e}-8$ & 0.133 \\
\hline 10 & $1024 \times 1024$ & 1.50 & $5.64 \mathrm{e}-3$ & $9.31 \mathrm{e}-9$ & $2.12 \mathrm{e}-8$ & $1.95 \mathrm{e}-8$ & 0.531 \\
\hline
\end{tabular}

In Table 8.1, the third column gives the total number $N_{i t}$ of equivalent iterations at level $n$. For instance, for $n=10$ and $4 \leq k \leq n, m_{k}$ iterations are required for the equation $B_{k} z_{n, k}=r_{k}^{0}$, where $m_{4}=15, m_{5}=13, m_{6}=8, m_{7}=5, m_{8}=2$, $m_{9}=1$, and $m_{10}=1$. By (8.2) we obtain

$$
N_{i t}=\sum_{k=4}^{n} \frac{m_{k}}{4^{n-k}} \approx 1.50
$$

The fourth column of the above table gives the initial residue, and the fifth column gives the residue when the algorithm terminates. Note that $\left\|r_{n}^{0}\right\|_{2}$ depends on $n$. But the first three digits of $\left\|r_{n}^{0}\right\|_{2}$ are the same for $n \geq 5$.

The sixth column gives the error $\left\|\Delta e_{n}\right\|_{2}=\left\|\Delta e_{n}\right\|_{L_{2}(\Omega)}$ of the approximate solution in the energy norm. For the purpose of comparison, in the seventh column we also list the discretization error $\left\|\Delta e_{n}^{*}\right\|_{2}=\left\|\Delta e_{n}^{*}\right\|_{L_{2}(\Omega)}$ in the energy norm. Recall that $e_{n}^{*}=u_{n}^{*}-u$ and $u_{n}^{*}=\mathcal{P}_{n} z_{n}^{*}$, where $z_{n}^{*}$ is the exact solution to the equation (7.6), which is obtained by sufficiently many iterations. We find

$$
\left\|\Delta e_{n}\right\|_{2} \leq 1.12\left\|\Delta e_{n}^{*}\right\|_{2} \text { for } 4 \leq n \leq 10 .
$$

This demonstrates that the approximate solution obtained by our algorithm achieves the level of discretization error. Moreover, we see that $\left\|\Delta e_{n+1}^{*}\right\|_{2} /\left\|\Delta e_{n}^{*}\right\|_{2}<$ $0.2506 \approx 1 / 4$ for $4 \leq n \leq 9$. Thus, for the energy norm, the computation indicates that the rate of convergence is of order 2, confirming the assertion made in Theorem 3.1.

The last column of the above table gives the CPU time in seconds for solving the linear system of equations $B_{n} z_{n}=\eta_{n}$. At level $n=10$, the matrix $B_{10}$ has size $1046529 \times 1046529$. Our algorithm takes only 0.531 of a second to solve the equation $B_{10} z_{10}=\eta_{10}$. 
Example 8.2. For $\left(x_{1}, x_{2}\right) \in \mathbb{R}^{2}$, let $z:=\left(x_{1}-1 / 2\right)^{2}+\left(x_{2}-1 / 2\right)^{2}-1 / 4$. Consider the biharmonic equation (1.4) on $\Omega$ with $f$ given by

$f\left(x_{1}, x_{2}\right)= \begin{cases}t\left[(4 z+1)^{2} \sin z+16(4 z+1)(1-\cos z)+32(z-\sin z)\right] & \text { if } z<0 \\ 0 & \text { if } z \geq 0\end{cases}$

where $t>0$ is so chosen that $\|f\|_{2}=1$. The exact solution of the equation is given by $u\left(x_{1}, x_{2}\right)=t\left[\sin z-z+z^{3} / 3\right]$ for $\left(x_{1}, x_{2}\right) \in \Omega$.

TABLE 8.2. Numerical results of Example 8.2

\begin{tabular}{|c|c|c|c|c|c|c|c|}
\hline Level $n$ & Grid $2^{n} \times 2^{n}$ & $N_{i t}$ & $\left\|r_{n}^{0}\right\|_{2}$ & $\left\|r_{n}\right\|_{2}$ & $\left\|\Delta e_{n}\right\|_{2}$ & $\left\|\Delta e_{n}^{*}\right\|_{2}$ & Time (s) \\
\hline 5 & $32 \times 32$ & 2.00 & $5.19 \mathrm{e}-3$ & $9.35 \mathrm{e}-6$ & $2.89 \mathrm{e}-5$ & $2.77 \mathrm{e}-5$ & 0.001 \\
\hline 6 & $64 \times 64$ & 1.81 & $5.19 \mathrm{e}-3$ & $2.32 \mathrm{e}-6$ & $7.47 \mathrm{e}-6$ & $6.78 \mathrm{e}-6$ & 0.002 \\
\hline 7 & $128 \times 128$ & 1.92 & $5.19 \mathrm{e}-3$ & $3.72 \mathrm{e}-7$ & $1.76 \mathrm{e}-6$ & $1.69 \mathrm{e}-6$ & 0.007 \\
\hline 8 & $256 \times 256$ & 1.60 & $5.19 \mathrm{e}-3$ & $1.35 \mathrm{e}-7$ & $4.50 \mathrm{e}-7$ & $4.21 \mathrm{e}-7$ & 0.026 \\
\hline 9 & $512 \times 512$ & 1.51 & $5.19 \mathrm{e}-3$ & $2.95 \mathrm{e}-8$ & $1.16 \mathrm{e}-7$ & $1.05 \mathrm{e}-7$ & 0.122 \\
\hline 10 & $1024 \times 1024$ & 1.43 & $5.19 \mathrm{e}-3$ & $9.61 \mathrm{e}-9$ & $2.84 \mathrm{e}-8$ & $2.63 \mathrm{e}-8$ & 0.523 \\
\hline
\end{tabular}

In this example, we have $\left\|\Delta e_{n}\right\|_{2} \leq 1.11\left\|\Delta e_{n}^{*}\right\|_{2}$ for $4 \leq n \leq 10$.

Example 8.3. Consider the biharmonic equation (1.4) with $f$ given by

$$
f\left(x_{1}, x_{2}\right)=t e^{\left(3 x_{1}-x_{2}\right)^{2}}, \quad\left(x_{1}, x_{2}\right) \in \Omega=(0,1)^{2},
$$

where $t>0$ is chosen so that $\|f\|_{2}=1$. In this case, the exact solution is unknown. Let $\tilde{e}_{n}:=u_{n}-u_{n+1}$ and $\tilde{e}_{n}^{*}:=u_{n}^{*}-u_{n+1}^{*}$.

TABLE 8.3. Numerical results of Example 8.3

\begin{tabular}{|c|c|c|c|c|c|c|c|}
\hline Level $n$ & Grid $2^{n} \times 2^{n}$ & $N_{i t}$ & $\left\|r_{n}^{0}\right\|_{2}$ & $\left\|r_{n}\right\|_{2}$ & $\left\|\Delta \tilde{e}_{n}\right\|_{2}$ & $\left\|\Delta \tilde{e}_{n}^{*}\right\|_{2}$ & Time (s) \\
\hline 5 & $32 \times 32$ & 2.75 & $1.15 \mathrm{e}-3$ & $7.02 \mathrm{e}-6$ & $3.58 \mathrm{e}-5$ & $3.58 \mathrm{e}-5$ & 0.001 \\
\hline 6 & $64 \times 64$ & 3.13 & $1.15 \mathrm{e}-3$ & $1.83 \mathrm{e}-6$ & $8.89 \mathrm{e}-6$ & $8.87 \mathrm{e}-6$ & 0.002 \\
\hline 7 & $128 \times 128$ & 3.25 & $1.15 \mathrm{e}-3$ & $4.20 \mathrm{e}-7$ & $2.20 \mathrm{e}-6$ & $2.20 \mathrm{e}-6$ & 0.009 \\
\hline 8 & $256 \times 256$ & 3.31 & $1.15 \mathrm{e}-3$ & $8.23 \mathrm{e}-8$ & $5.51 \mathrm{e}-7$ & $5.51 \mathrm{e}-7$ & 0.048 \\
\hline 9 & $512 \times 512$ & 2.27 & $1.15 \mathrm{e}-3$ & $4.98 \mathrm{e}-8$ & $1.38 \mathrm{e}-7$ & $1.38 \mathrm{e}-7$ & 0.157 \\
\hline
\end{tabular}


Example 8.4. Let $\left(c_{i_{1}, i_{2}}\right)_{0 \leq i_{1}, i_{2}<2^{10}}$ be a random array of real numbers between 0 and 1. Consider the biharmonic equation (1.4) with $f$ being a piecewise constant function given by

$$
f\left(x_{1}, x_{2}\right):=t c_{i_{1}, i_{2}} \text { for } \frac{i_{1}}{2^{10}}<x_{1}<\frac{i_{1}+1}{2^{10}} \text { and } \frac{i_{2}}{2^{10}}<x_{2}<\frac{i_{2}+1}{2^{10}}
$$

where $t>0$ is chosen so that $\|f\|_{2}=1$.

TABLE 8.4. Numerical results of Example 8.4

\begin{tabular}{|c|c|c|c|c|c|c|c|}
\hline Level $n$ & Grid $2^{n} \times 2^{n}$ & $N_{i t}$ & $\left\|r_{n}^{0}\right\|_{2}$ & $\left\|r_{n}\right\|_{2}$ & $\left\|\Delta \tilde{e}_{n}\right\|_{2}$ & $\left\|\Delta \tilde{e}_{n}^{*}\right\|_{2}$ & Time (s) \\
\hline 5 & $32 \times 32$ & 2.75 & $5.55 \mathrm{e}-3$ & $8.78 \mathrm{e}-6$ & $3.31 \mathrm{e}-5$ & $3.31 \mathrm{e}-5$ & 0.001 \\
\hline 6 & $64 \times 64$ & 3.06 & $5.55 \mathrm{e}-3$ & $2.01 \mathrm{e}-6$ & $8.29 \mathrm{e}-6$ & $8.29 \mathrm{e}-6$ & 0.002 \\
\hline 7 & $128 \times 128$ & 1.98 & $5.55 \mathrm{e}-3$ & $9.55 \mathrm{e}-7$ & $2.08 \mathrm{e}-6$ & $2.08 \mathrm{e}-6$ & 0.006 \\
\hline 8 & $256 \times 256$ & 1.99 & $5.55 \mathrm{e}-3$ & $2.04 \mathrm{e}-7$ & $5.35 \mathrm{e}-7$ & $5.35 \mathrm{e}-7$ & 0.032 \\
\hline 9 & $512 \times 512$ & 1.93 & $5.55 \mathrm{e}-3$ & $6.20 \mathrm{e}-8$ & $1.42 \mathrm{e}-7$ & $1.42 \mathrm{e}-7$ & 0.139 \\
\hline
\end{tabular}

The biharmonic equation has been extensively studied in the literature. For numerical solutions of the biharmonic equation, the finite difference method was used in [7, 6], and [1, and the finite element method was employed in [32, 27, and [28. Chang, Wong, Fu in [7, and Chang and Huang in [6] used difference schemes of second order, while Altas, Dym, Gupta and Manohar in [1] introduced a vector difference scheme of fourth-order in their solutions of the biharmonic equation. Sun in 32 and Oswald in 27] considered preconditioning techniques for the biharmonic equation discretized by quadratic and cubic splines, respectively. Silvester and Mihajlović in 28 proposed a multigrid preconditioning operator for the decoupled equation (7.5).

All the papers mentioned above, except [1, focused on residue reduction for the preconditioned matrices. For relative residue reduction in the $\ell_{2}$ norm, the above numerical examples show that our algorithm requires considerably fewer iterations than those reported in [32] and [27. Let us discuss relative residue reduction in the $\ell_{\infty}$ norm given by the quantity $\left\|r_{n}\right\|_{\infty} /\left\|r_{n}^{0}\right\|_{\infty}$. In Table 8.5 , for $\varepsilon=10^{-4}, 10^{-5}$, and $10^{-6}$, we list the average number of iterations needed for $\tau_{n}:=\left\|r_{n}\right\|_{\infty} /\left\|r_{n}^{0}\right\|_{\infty}<\varepsilon$ in the above four examples:

TABLE 8.5. Relative residue reduction

\begin{tabular}{|c|c|c|c|c|}
\hline Level $n$ & Grid $2^{n} \times 2^{n}$ & $\tau_{n}<10^{-4}$ & $\tau_{n}<10^{-5}$ & $\tau_{n}<10^{-6}$ \\
\hline 8 & $256 \times 256$ & 1.8 & 3.0 & 5.0 \\
\hline 9 & $512 \times 512$ & 1.4 & 2.0 & 2.9 \\
\hline 10 & $1024 \times 1024$ & 1.3 & 1.5 & 1.9 \\
\hline
\end{tabular}


The relative residue reduction in the $\ell_{\infty}$ norm was discussed in 28, for the $258 \times 258$ grid discretized by piecewise linear elements. It required 4 BICGSTAB iterations to get $\tau_{8}<6.2 \times 10^{-4}$ and 20 BICGSTAB iterations to get $\tau_{8}<1.2 \times 10^{-6}$. Further, 3 multigrid $V(1,1)$ cycles per itearation were performed for preconditioning (see Table 4 iii) in [28). The algebraic multigrid method was used [7] and [6]. It was reported in Table 10 of [6] that more than 40 iterations were needed for the relative residue reduction in the $\ell_{\infty}$ norm to be less than $10^{-6}$.

We remark that residue reductions are not fully comparable, because the corresponding matrices are different in different contexts. We think that it is more appropriate to compare the efficiency of numerical algorithms to achieve the level of discretization error. The wavelet method we propose has the advantage that the number of iterations needed to achieve the level of discretization error will not increase as the mesh size decreases. Thus, the wavelet method is suitable for large-scale computation. In comparison, in most of the aforementioned papers, the number of iterations would increase as the mesh size decreases.

\section{Numerical examples: ERror EStimates in the $L_{2}$ AND $L_{\infty}$ NORMS}

In this section we investigate numerical solutions of the biharmonic equation and estimate errors of approximate solutions in the $L_{2}$ and $L_{\infty}$ norms. We simply perform more iterations to achieve the level of discretization error. For the examples considered in this section, 7 equivalent PCG iterations based on our wavelets will be sufficient.

The following example was considered in $[1$. We define

$$
\left\|e_{n}\right\|_{\infty}:=\max \left\{\left|e_{n}\left(i_{1} / 2^{n}, i_{2} / 2^{n}\right)\right|: 0 \leq i_{1}, i_{2} \leq 2^{n}\right\} .
$$

This definition agrees with the one given in [1].

Example 9.1. Consider the biharmonic equation (1.4) on $\Omega=(0,1)^{2}$ with $f$ given by

$$
f\left(x_{1}, x_{2}\right)=16 \pi^{4}\left[4 \cos \left(2 \pi x_{1}\right) \cos \left(2 \pi x_{2}\right)-\cos \left(2 \pi x_{1}\right)-\cos \left(2 \pi x_{2}\right)\right], \quad\left(x_{1}, x_{2}\right) \in \Omega .
$$

The exact solution of the equation is

$$
u\left(x_{1}, x_{2}\right)=\left[1-\cos \left(2 \pi x_{1}\right)\right]\left[1-\cos \left(2 \pi x_{2}\right)\right], \quad\left(x_{1}, x_{2}\right) \in \Omega .
$$

The numerical results are listed in Table 9.1.

TABLE 9.1. Error estimates in the maximum norm

\begin{tabular}{|c|c|c|c|c|c|c|}
\hline Level $n$ & Grid $2^{n} \times 2^{n}$ & $\left\|e_{n}^{D}\right\|_{\infty}$ & Time (s) & $\left\|e_{n}^{L D}\right\|_{\infty}$ & Time (s) & $\left\|e_{n}^{*}\right\|_{\infty}$ \\
\hline 5 & $32 \times 32$ & $6.28 \mathrm{e}-6$ & 0.003 & $6.28 \mathrm{e}-6$ & 0.004 & $6.28 \mathrm{e}-6$ \\
\hline 6 & $64 \times 64$ & $3.90 \mathrm{e}-7$ & 0.007 & $3.90 \mathrm{e}-7$ & 0.013 & $3.90 \mathrm{e}-7$ \\
\hline 7 & $128 \times 128$ & $2.44 \mathrm{e}-8$ & 0.026 & $2.44 \mathrm{e}-8$ & 0.047 & $2.44 \mathrm{e}-8$ \\
\hline 8 & $256 \times 256$ & $4.19 \mathrm{e}-9$ & 0.110 & $1.52 \mathrm{e}-9$ & 0.198 & $1.52 \mathrm{e}-9$ \\
\hline 9 & $512 \times 512$ & $4.27 \mathrm{e}-8$ & 0.507 & $8.34 \mathrm{e}-11$ & 0.885 & $8.31 \mathrm{e}-11$ \\
\hline
\end{tabular}


In Table 9.1 , the third column gives the error $\left\|e_{n}^{D}\right\|_{\infty}$ by using the double precision arithmetic, and the fourth column gives the corresponding CPU time in seconds. Moreover, the fifth column gives the error $\left\|e_{n}^{L D}\right\|_{\infty}$ by using the long double precision arithmetic, and the sixth column gives the corresponding CPU time in seconds. Finally, the last column gives the discretization error $\left\|e_{n}^{*}\right\|_{\infty}$ in the $\ell_{\infty}$ norm.

We observe that, starting from level 8 , the accuracy of the approximate solutions is affected by the roundoff errors if the double precision arithmetic is used. But the long double precision gives the desired accuracy at levels 8 and 9 . We also observe that $\left\|e_{n}^{*}\right\|_{\infty} /\left\|e_{n+1}^{*}\right\|_{\infty}<0.626 \approx 2^{-4}$ for $n=5,6,7,8$. Hence, the rate of convergence is of order 4.

A vector difference scheme of order 4 was used in [1]. The matrix obtained from discretization using their scheme has size $3\left(2^{n}-1\right)^{2} \times 3\left(2^{n}-1\right)^{2}$ at level $n$. In comparison, the matrix $B_{n}$ has size $\left(2^{n}-1\right)^{2} \times\left(2^{n}-1\right)^{2}$. But our discretization error $\left\|e_{n}^{*}\right\|_{\infty}$ is smaller. For instance, for $n=7$ we have $\left\|e_{7}^{*}\right\|_{\infty} \approx 2.44 \times 10^{-8}$, while the corresponding discretization error in [1] is $4.2 \times 10^{-8}$. It was reported in 1 that 3 FMG (Full Mutligrid) $W(3,2)$-cycles were used to achieve the level of discretization error. We estimate that a multiplication of their matrix with a vector costs twice as much as a multiplication of our matrix $\left(B_{n}\right)$ with a vector (see the above comparison of the matrix size). Consequently, we estimate that a multigrid $V(3,2)$-cycle costs as much as 5 PCG iterations of our scheme. The computational cost of a FMG $W(3,2)$-cycle is about twice the cost of a simple $V(3,2)$-cycle (see [1]). Thus, the computational cost of 3 FMG $W(3,2)$-cycles is about the cost of 30 PCG iterations of our scheme.

Example 9.2. Consider the biharmonic equation (1.4) on $\Omega$ with $f$ given by

$$
f\left(x_{1}, x_{2}\right)=e^{\left(3 x_{1}-x_{2}\right)^{2}}, \quad\left(x_{1}, x_{2}\right) \in \Omega .
$$

In this case, the exact solution is unknown. Recall that $\tilde{e}_{n}=u_{n}-u_{n+1}$ and $\tilde{e}_{n}^{*}=$ $u_{n}^{*}-u_{n+1}^{*}$. In Table 9.2 we list numerical results of the approximate solutions that achieve the level of discretization error in the $L_{2}$ norm. The numerical computation clearly shows that the rate of convergence is of order 4 , confirming the conclusion of Theorem 3.1 .

TABLE 9.2. Error estimates in the $L_{2}$ norm

\begin{tabular}{|c|c|c|c|c|c|c|}
\hline Level $n$ & Grid $2^{n} \times 2^{n}$ & $\left\|\tilde{e}_{n}^{D}\right\|_{2}$ & Time (s) & $\left\|\tilde{e}_{n}^{L D}\right\|_{2}$ & Time (s) & $\left\|\tilde{e}_{n}^{*}\right\|_{2}$ \\
\hline 5 & $32 \times 32$ & $7.18 \mathrm{e}-7$ & 0.003 & $7.18 \mathrm{e}-7$ & 0.004 & $7.18 \mathrm{e}-7$ \\
\hline 6 & $64 \times 64$ & $4.18 \mathrm{e}-8$ & 0.007 & $4.18 \mathrm{e}-8$ & 0.013 & $4.18 \mathrm{e}-8$ \\
\hline 7 & $128 \times 128$ & $2.54 \mathrm{e}-9$ & 0.026 & $2.54 \mathrm{e}-9$ & 0.047 & $2.54 \mathrm{e}-9$ \\
\hline 8 & $256 \times 256$ & $1.67 \mathrm{e}-10$ & 0.110 & $1.59 \mathrm{e}-10$ & 0.198 & $1.58 \mathrm{e}-10$ \\
\hline 9 & $512 \times 512$ & $1.20 \mathrm{e}-9$ & 0.507 & $1.00 \mathrm{e}-11$ & 0.885 & $9.92 \mathrm{e}-12$ \\
\hline
\end{tabular}




\section{Fourth-ORDER ELLIPTIC EQUATIONS}

In this section we extend our study to general elliptic equations of fourth-order. If the Dirichlet form of a fourth-order elliptic operator is strictly coercive, then the wavelet bases constructed in $\S 6$ are still applicable to numerical solutions of the corresponding fourth-order elliptic equation with the homogeneous boundary conditions. For simplicity, let us consider the following elliptic equation:

$$
\begin{cases}\Delta(a(x) \Delta u)(x)=f(x) & \text { for } x \in \Omega, \\ u(x)=\frac{\partial u}{\partial n}(x)=0 & \text { for } x \in \partial \Omega,\end{cases}
$$

where $a(x)=a\left(x_{1}, x_{2}\right)$ is a continuous function on $\Omega$ and there exist two positive constants $K_{1}$ and $K_{2}$ such that $K_{1} \leq a\left(x_{1}, x_{2}\right) \leq K_{2}$ for all $\left(x_{1}, x_{2}\right) \in \Omega$. Consequently, there exist two positive constants $C_{1}$ and $C_{2}$ such that

$$
C_{1}|u|_{H_{0}^{2}(\Omega)}^{2} \leq\langle a \Delta u, \Delta u\rangle \leq C_{2}|u|_{H_{0}^{2}(\Omega)}^{2} \quad \forall u \in H_{0}^{2}(\Omega) .
$$

The variational form corresponding to the preceding elliptic equation is

$$
\langle a \Delta u, \Delta v\rangle=\langle f, v\rangle \quad \forall v \in H_{0}^{2}(\Omega) .
$$

Thus, our wavelet bases can be used to discretize the above equation. We give two examples of numerical computation as follows. The long double precision arithmetic will be used in the computation.

Example 10.1. Let $a\left(x_{1}, x_{2}\right):=\left(1+x_{1}\right)\left(1+x_{2}\right)$ for $\left(x_{1}, x_{2}\right) \in \Omega=(0,1)^{2}$. Suppose that $f(x)=\Delta(a(x) \Delta u)(x)$ for $x \in \Omega$, where $u$ is given by

$$
u\left(x_{1}, x_{2}\right)=\left[1-\cos \left(2 \pi x_{1}\right)\right]\left[1-\cos \left(2 \pi x_{2}\right)\right] / 4, \quad\left(x_{1}, x_{2}\right) \in \Omega .
$$

Then $u$ is the exact solution of (1.4). The numerical results are listed in Table 10.1.

TABLE 10.1. Numerical results of Example 10.1

\begin{tabular}{|c|c|c|c|c|}
\hline Level $n$ & Grid $2^{n} \times 2^{n}$ & $\left\|e_{n}\right\|_{2}$ & $\left\|e_{n}^{*}\right\|_{2}$ & Time (s) \\
\hline 5 & $32 \times 32$ & $7.38 \mathrm{e}-7$ & $7.38 \mathrm{e}-7$ & 0.017 \\
\hline 6 & $64 \times 64$ & $4.57 \mathrm{e}-8$ & $4.57 \mathrm{e}-8$ & 0.053 \\
\hline 7 & $128 \times 128$ & $2.85 \mathrm{e}-9$ & $2.85 \mathrm{e}-9$ & 0.196 \\
\hline 8 & $256 \times 256$ & $1.78 \mathrm{e}-10$ & $1.78 \mathrm{e}-10$ & 0.806 \\
\hline 9 & $512 \times 512$ & $1.10 \mathrm{e}-11$ & $1.10 \mathrm{e}-11$ & 3.524 \\
\hline
\end{tabular}

Example 10.2. Let $a\left(x_{1}, x_{2}\right):=1+0.5 \sin \left[10.8\left(x_{1}-x_{2}\right)\right]$ for $\left(x_{1}, x_{2}\right) \in \Omega=(0,1)^{2}$. Let $f(x)=\Delta(a(x) \Delta u)(x)$ for $x \in \Omega$, where $u$ is given by (10.2). Then $u$ is the exact solution of (10.1). The numerical results are listed in Table 10.2. 
TABLE 10.2. Numerical results of Example 10.2

\begin{tabular}{|c|c|c|c|c|}
\hline Level $n$ & Grid $2^{n} \times 2^{n}$ & $\left\|e_{n}\right\|_{2}$ & $\left\|e_{n}^{*}\right\|_{2}$ & Time (s) \\
\hline 5 & $32 \times 32$ & $8.25 \mathrm{e}-7$ & $8.25 \mathrm{e}-7$ & 0.017 \\
\hline 6 & $64 \times 64$ & $5.11 \mathrm{e}-8$ & $5.11 \mathrm{e}-8$ & 0.053 \\
\hline 7 & $128 \times 128$ & $3.21 \mathrm{e}-9$ & $3.18 \mathrm{e}-9$ & 0.196 \\
\hline 8 & $256 \times 256$ & $2.04 \mathrm{e}-10$ & $1.99 \mathrm{e}-10$ & 0.806 \\
\hline 9 & $512 \times 512$ & $1.26 \mathrm{e}-11$ & $1.21 \mathrm{e}-11$ & 3.524 \\
\hline
\end{tabular}

\section{REFERENCES}

[1] I. Altas, J. Dym, M. M. Gupta, and R. P. Manohar, Multigrid solution of automatically generated high-order discretizations for the biharmonic equation, SIAM J. Sci. Comput. 19 (1998), 1575-1585. MR1618733

[2] H. Blum and R. Rannacher, On the boundary value problem of the biharmonic operator on domains with angular corners, Math. Mech. Appl. Sci. 2 (1980), 556-581. MR595625 (82a:35022)

[3] C. de Boor, A Practical Guide to Splines, Springer-Verlag, New York, 1978. MR507062 (80a:65027)

[4] C. de Boor and G. Fix, Spline approximation by quasi-interpolants, J. Approx. Theory 8 (1973), 19-45. MR0340893 (49:5643)

[5] S. C. Brenner and L. R. Scott, The Mathematical Theory of Finite Element Methods, Second Edition, Springer-Verlag, New York, 2002. MR1894376 (2003a:65103)

[6] Q. S. Chang and Z. H. Huang, Efficient algebraic multigrid algorithms and their convergence, SIAM J. Sci. Comput. 24 (2002), 597-618. MR1951057(2004m:65035)

[7] Q. S. Chang. Y. S. Wong, and H. Q. Fu, On the algebraic multigrid method, Journal of Computational Physics 125 (1996), 279-292. MR.1388150 (96m:65089)

[8] C. K. Chui and J. Z. Wang, On compactly supported spline wavelets and a duality principle, Trans. Amer. Math. Soc. 330 (1992), 903-916. MR:1076613 (92f:41020)

[9] W. Dahmen, A. Kunoth, and K. Urban, Biorthogonal spline wavelets on the interval stability and moment conditions, Applied and Computational Harmonic Analysis 6 (1999), 132-196. MR.1676771 (99m:42046)

[10] O. Davydov and R. Stevenson, Hierachical Riesz bases for $H^{s}(\Omega), 1<s<5 / 2$, Constructive Approximation 22 (2005), 365-394. MR2164141 (2006d:41006)

[11] R. DeVore, B. Jawerth, and V. Popov, Compression of wavelet decompositions, Amer. J. Math. 114 (1992), 737-785. MR.1175690 (94a:42045)

[12] L. C. Evans, Partial Differential Equations, Graduate Studies in Mathematics, Volume 19, Amer. Math. Soc., Providence, Rhode Island, 1998. MR.1625845 (99e:35001)

[13] B. Han and Q. T. Jiang, Multiwavelets on the interval, Applied and Computational Harmonic Analysis 12 (2002), 100-127. MR.1874917 (2002k:65233)

[14] B. Han and Z. W. Shen, Wavelets with short support, SIAM J. Math. Anal. 38 (2006), 530-556. MR2237160 (2007c:42051)

[15] R. A. Horn and C. R. Johnson, Topics in Matrix Analysis, Cambridge University Press, Cambridge, 1991. MR1091716 (92e:15003)

[16] R. Q. Jia, Approximation with scaled shift-invariant spaces by means of quasi-projection operators, Journal of Approximation Theory 131 (2004), 30-46. MR2103832 (2005h:41035)

[17] R. Q. Jia, Bessel sequences in Sobolev spaces, Applied and Computational Harmonic Analysis 20 (2006), 298-311. MR2207841 (2007a:42067)

[18] R. Q. Jia, Spline wavelets on the interval with homogeneous boundary conditions, Advances in Computational Mathematics 30 (2009), 177-200. MR2471447 (2009k:42073) 
[19] R. Q. Jia, Approximation by quasi-projection operators in Besov spaces, Journal of Approximation Theory 162 (2010), 186-200. MR.2565832

[20] R. Q. Jia and S. T. Liu, Wavelet bases of Hermite cubic splines on the interval, Advances in Computational Mathematics 25 (2006), 23-39. MR.2231693 (2007e:42043)

[21] R. Q. Jia and S. T. Liu, $C^{1}$ spline wavelets on triangulations, Mathematics of Computation 77 (2008), 287-312. MR2353954 (2008m:41011)

[22] R. Q. Jia, J. Z. Wang, and D. X. Zhou, Compactly supported wavelet bases for Sobolev spaces, Applied and Computational Harmonic Analysis 15 (2003), 224-241. MR2010944 (2004h:42042)

[23] J. J. Lei, R. Q. Jia, and E. W. Cheney, Approximation from shift-invariant spaces by integral operators, SIAM J. Math. Anal. 28 (1997), 481-498. MR1434046 (98h:41026)

[24] R. J. LeVeque, Finite Difference Methods for Ordinary and Partial Differential Equations, SIAM, Philadelphia, 2007. MR2378550(2009a:65173)

[25] S. Li and J. Xian, Biorthogonal multiple wavelets generated by vector refinement equation, Science in China Series A: Mathematics 50 (2007), 1015-1025. MR2355873 (2008i:42071)

[26] Y. Meyer, Wavelets and Operators, Cambridge University Press, Cambridge, 1992. MR.1228209 (94f:42001)

[27] P. Oswald, Multilevel preconditioners for discretizations of the biharmonic equation by rectangular finite elements, Numerical Linear Algebra with Applications 2 (1995), 487-505. MR1367057 (97c:65206)

[28] D. J. Silvester and M. D. Mihajlović, Efficient preconditioning of the biharmonic equation, Numerical Analysis Report No. 362 (2000), Manchester Center for Computational Mathematics, University of Manchester.

[29] E. M. Stein, Singular Integrals and Differentiability Properties of Functions, Princeton University Press, Princeton, New Jersey, 1970. MR0290095 (44:7280)

[30] R. Stevenson, Stable three-point wavelet bases on general meshes, Numer. Math. 80 (1998), 131-158. MR1642527 (2000d:65221)

[31] G. Strang and G. Fix, An Analysis of the Finite Element Method, Prentice-Hall, Englewood Cliffs, New Jersey, 1973. MR.0443377 (56:1747)

[32] J. C. Sun, Domain decompositions and multilevel PCG method for solving 3-D fourth order problems, Contemporary Mathematics 157 (1994), 71-78. MR1262607 (94j:65133)

[33] P. S. Vassilevski and J.-P. Wang, Stabilizing the hierarchical bases by approximate wavelets, I: Theory, Numer. Lin. Alg. Appl. 4 (1997), 103-126. MR.1443598 (98c:65197)

[34] R. M. Young, An Introduction to Nonharmonic Fourier Series, Academic Press, New York, 1980. MR591684 (81m:42027)

Department of Mathematical and Statistical Sciences, University of Alberta, EdMONTON, Canada T6G 2G1

E-mail address: rjia@ualberta.ca

Department of Mathematics, McMaster University, 1280 Main Street West, Hamilton, Ontario, Canada L8S 4K1

E-mail address: wzhao@math.mcmaster.ca 